\section{Catalytic Enolate Arylation with 3-Bromoindoles Allows the Formation of $\beta$-Carbolines}

\author{
C. Henrique Alves Esteves, ${ }^{\mathrm{a}}$ Peter D. Smith ${ }^{\mathrm{b}}$ and Timothy J. \\ Donohoe $^{\mathrm{a} *}$ \\ ${ }^{a}$ Department of Chemistry, University of Oxford, Chemistry \\ Research Laboratory, Mansfield Road, Oxford, OX1 3TA, UK.
}

${ }^{b}$ AstraZeneca, Pharmaceutical Sciences, Silk Road Business Park, Macclesfield, SK10 2NA, UK.

timothy.donohoe@chem.ox.ac.uk

\begin{abstract}
RECEIVED DATE (will be automatically inserted after manuscript is accepted).
\end{abstract}

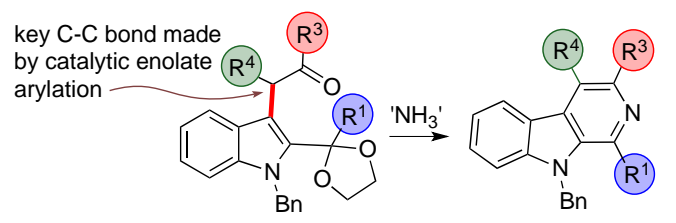

The synthesis of substituted $\beta$-carbolines was accomplished by utilizing the catalytic enolate arylation reaction of ketones in conjunction with several 3-bromoindole derivatives. Quenching of the arylation reaction in situ with an electrophile allowed ready incorporation of functionality at the carboline C-4 position in an efficient one-pot protocol.

$\beta$-Carbolines are important $N$-heterocyclic aromatic compounds that have been extensively studied for their wideranging bioactivity. ${ }^{1}$ These compounds are also widely found in natural products extracted from various sources, such as the dichotomides, ${ }^{2}$ from the roots of Stellaria dichotoma; the metatacarbolines, ${ }^{3}$ from the fruiting bodies of Mycena metata; hirsutaside $\mathrm{D},{ }^{4}$ from the leaves of Uncaria hirsute, hyrtiocarboline, ${ }^{5}$ from the marine sponge Hyrtios reticulatus, and stolonines, ${ }^{6}$ from the marine tunicate Cnemidocarpa stolonifera.

Some of the recently reported methodologies to prepare $\beta$ carbolines involve the metal-catalyzed iminoannulation of alkynes, ${ }^{7}$ the Rh-catalyzed cycloadditon of yne-ynamides with methylcyanoformate, ${ }^{8}$ cascade annulation of alkynols, ${ }^{9}$ photocyclization of anilinohalopyridines,,$^{10}$ coupling of anilines and halopyridines, ${ }^{11}$ Pd-catalyzed ring-expansion of azidoalcohols $^{12}$ and cyclization of vinylindoles. ${ }^{13}$ While impressive, the main limitations of these methods lie in the formation of regioisomers or the limited accessibility of a variety of substitution patterns in the heterocyclic ring.

We sought a route to these heterocycles that would allow the introduction of many different groups onto the aromatic nucleus but without the complications arising from the formation of regioisomers. In this regard, our approach to the synthesis of carbolines relies on the use of catalytic enolate arylation reaction of ketones, ${ }^{14}$ a powerful catalytic reaction still underused in the synthesis of aromatic heterocycles. ${ }^{15}$ Previous work from our group has shown that ketones can be easily arylated by reaction of substituted aryl or vinyl halides so that the products are ideally configured (masked 1,4dicarbonyls) for an aromatization step to furnish substituted isoquinolines ${ }^{16}$ or pyridines ${ }^{17}$ (Figure 1a).

Herein, we have chosen a 3-bromoindole partner $\mathbf{A}$ for the enolate arylation (Figure 1b). ${ }^{18}$ The installation of a protected carbonyl at C-2 of the indole bromide partner would provide the functionality required to aromatize the ring after arylation was complete (see $\mathbf{B} \rightarrow \mathbf{C}$ ). Moreover, from previous precedent, ${ }^{16,19}$ we anticipated that an ability to add an electrophile directly to the catalytic arylation reaction would allow extra functionalization to be added to the product (see $\mathrm{R}^{4}$ in $\mathrm{B}$ ) that would ultimately allow derivatisation at the $\mathrm{C}-4$ position of the carboline without the addition of extra synthetic steps.

Figure 1: Catalytic enolate arylation route to $\beta$-carbolines
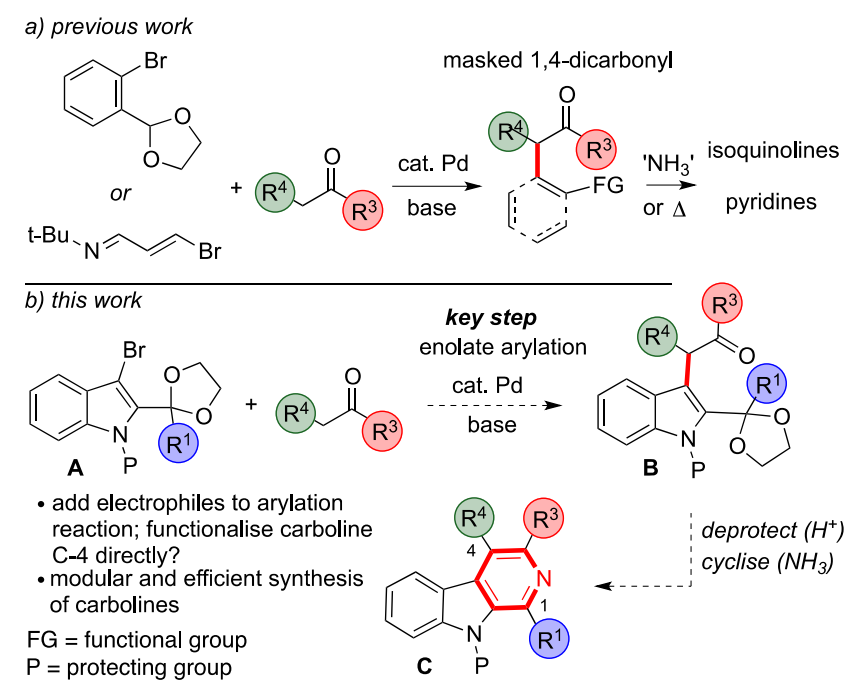

Initially, we selected 1-benzyl-3-bromo-2-(1,3-dioxolan-2yl)- $1 H$-indole (1) and acetophenone (2) for optimization studies using catalytic palladium (Table 1). Compound 1 was made in high yield from the precursor indole-2carboxaldehyde via reaction with NBS, followed by $\mathrm{N}$ benzylation with base and $\mathrm{BnBr}$ and then acetal formation with ethylene glycol and $p$ TSA. $^{20}$

We began our screening by treating $\mathbf{1}$ and acetophenone (2) with catalytic $\mathrm{Pd}(\mathrm{d} t \mathrm{bpf}) \mathrm{Cl}_{2}{ }^{21}$ and $\mathrm{NaO} t \mathrm{Bu}$ in THF (Table 1), which are conditions previously used for related arylations in our laboratory, ${ }^{16}$ and they afforded product $\mathbf{3}$ in an encouraging $52 \%$ yield (entry 1). Pleasingly, compound 3 could be isolated in $93 \%$ yield when the $\mathrm{NaO} t \mathrm{Bu}$ base was replaced by LiHMDS (entry 2 ).

Further studies showed that the arylation was tolerant of changes to the reaction parameters. For example, lower catalyst loadings and/or ketone equivalents were examined, and these delivered the desired product in good yields (entries $3-5)$. Moreover, a reasonable yield of 3 (66\%) was obtained 
when the arylation reaction was carried out at room temperature (entry 6). Finally, a different catalyst was screened with $\mathrm{Pd}(\text { amphos })_{2} \mathrm{Cl}_{2}$, (this was also previously very active in our hands) delivering the product in a disappointing $17 \%$ yield (entry 7 ).

Table 1: Optimization Studies for the Pd-Catalyzed $\alpha$-Arylation of 1

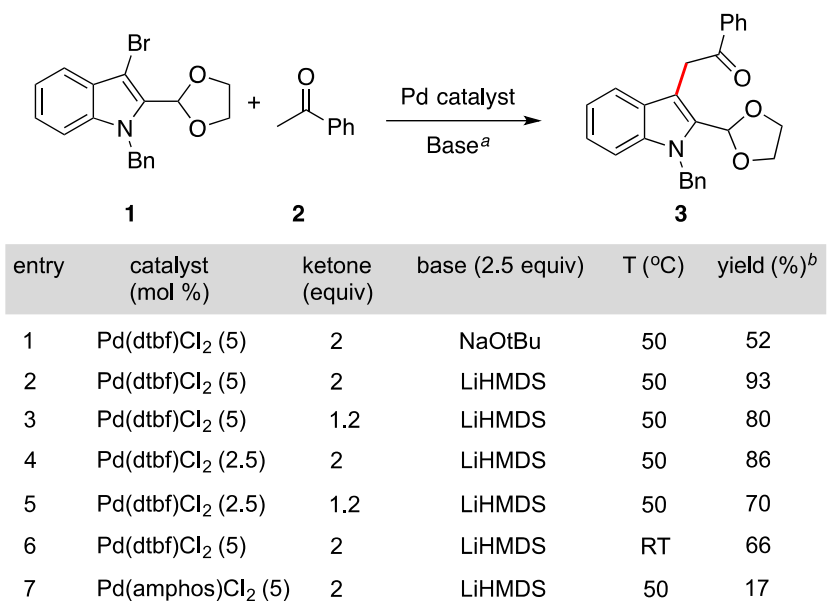

${ }^{a}$ Indole 1 (1.0 eq.),THF, $24 \mathrm{~h}$

${ }^{b}$ Isolated yields

Pleasingly, the reaction of arylated product 3 with ammonium chloride in ethanol/water was sufficiently acidic to deprotect the acetal and allow cyclisation to give the corresponding carboline 4 in $94 \%$ yield (Scheme 1), thus validating our approach to these aromatic heterocycles. Moreover, the N-benzyl group was easily removed from the carboline products using $\mathrm{AlCl}_{3}(\mathbf{4} \rightarrow \mathbf{5}$ in $88 \%$ yield $){ }^{22}$

Scheme 1: Cyclization of 3 to furnish $\beta$-carboline 4

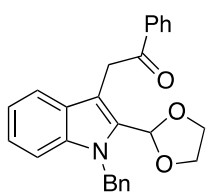

3

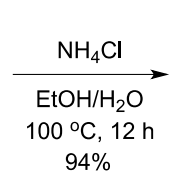

$94 \%$

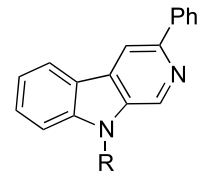

$\begin{array}{ll}\mathbf{4}(\mathrm{R}=\mathrm{Bn}) & \begin{array}{c}\mathrm{AlCl}_{3} \\ \text { toluene, } \mathrm{RT} \\ 8(\mathrm{R}=\mathrm{H})\end{array}\end{array}$
The next stage involved an exploration of the substrate scope, and in order to do this we prepared more substituted indole bromides to be coupled with a selection of commercially available ketones (Scheme 2). Our synthesis of the requisite indole bromides began from readily available compound $6,{ }^{23}$ which was N-benzylated (7) and then derivatised into two different ketones $(\mathbf{8}, \mathbf{9})$ via reaction with a Grignard reagent. After bromination at C-3 (10, 11), ketone 10 was protected as an acetal (12) under standard conditions.

Scheme 2: Synthesis of substituted indole bromides
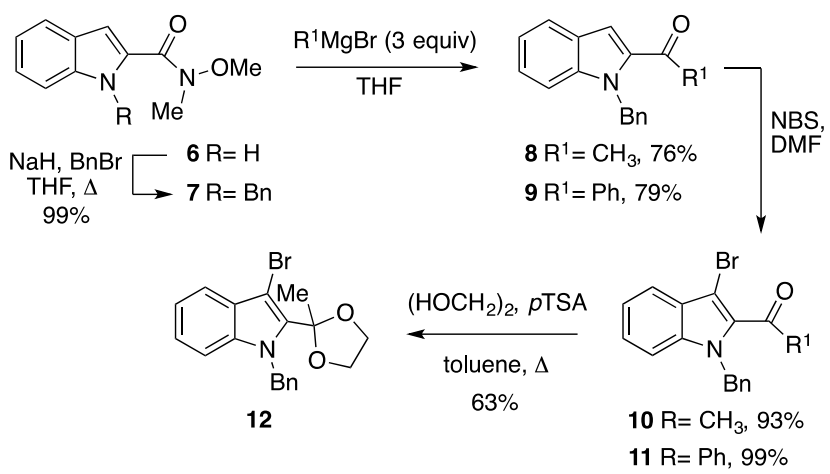

With the key compounds in hand, we were able to explore the substrate scope, and Table 2 shows a set of carbolines (4, $14,16,18)$ that were prepared via this two-step sequence of enolate arylation, using the optimized conditions from Table 1 , followed by aromatization. Use of this methodology allowed substitution at the carboline C-1, C-3 and C-4 positions simply by using a substituted ketone or altering the substitution pattern of the ketal protected indole bromide in the arylation step. Note that some groups (eg $\mathrm{Ph}$ ) could be introduced at the carboline $\mathrm{C}-1$ position without the need for ketal protection (see $\mathbf{1 1} \rightarrow \mathbf{1 7} \rightarrow \mathbf{1 8}$ ); in this case the conjugated nature of the ketone at $\mathrm{C}-2$ of the indole makes it unreactive to competing aldol type side reactions.

Table 2: Synthesis of substituted $\beta$-carbolines in two steps 

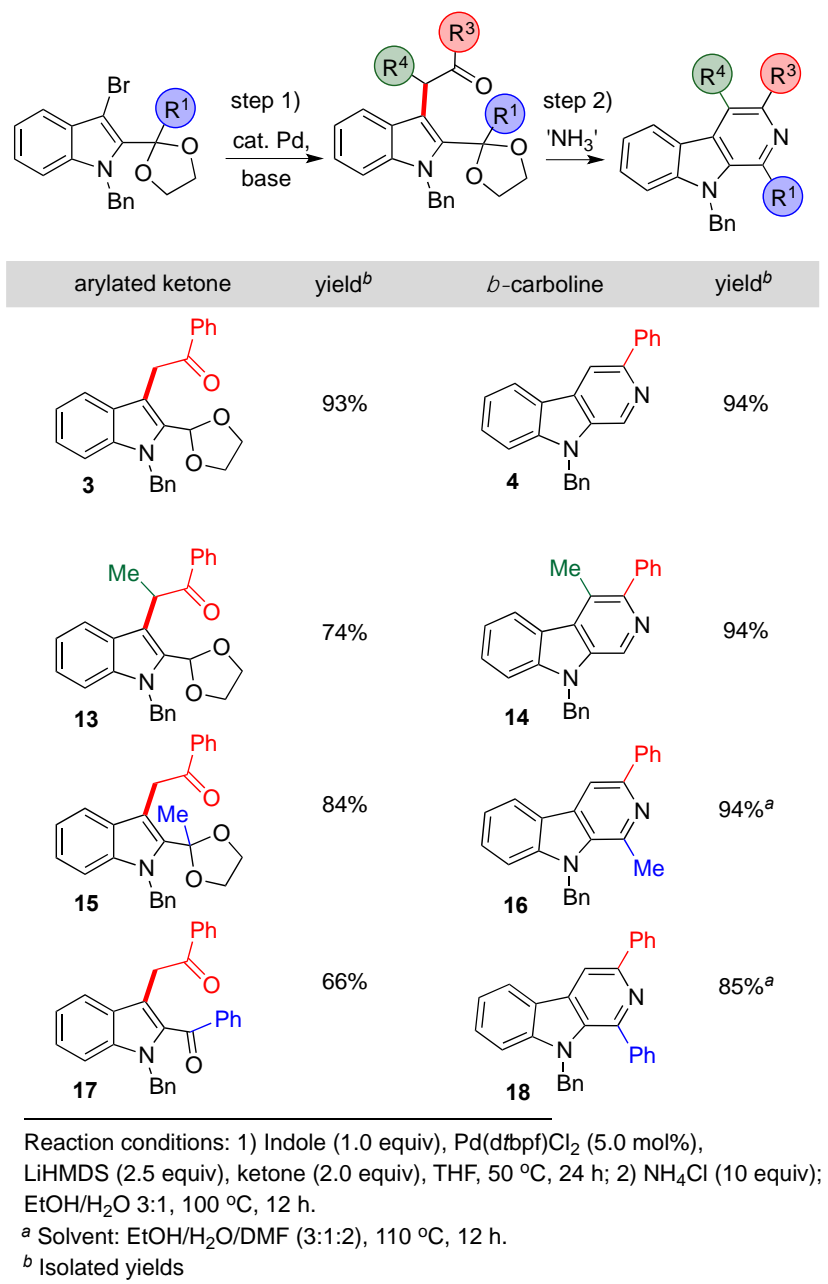

After these positive results we then sought to develop a onepot procedure for the arylation/aromatization sequence. This worked well and starting from $\mathbf{1}$ and $\mathbf{2}$ we could quench the arylation reaction directly with acid and then ammonia to furnish carboline 4 in $68 \%$ yield for the one-pot process (Table 3). The one-pot sequence was optimal when it began with an enolate arylation, followed by the sequential addition of acid (to hydrolyse the acetal), neutralization $\left(\mathrm{NaHCO}_{3}\right)$ and then addition of ammonia $(7 \mathrm{M}$ in $\mathrm{MeOH})$ to perform the aromatization.

However, as we switched to more substituted ketone substrates in order to expand the methodology, we found the arylation conditions that had proved optimal for acetophenone in Table 1 showed poor conversion (see $\mathbf{1 9}$ and $\mathbf{2 0}$ with $34 \%$ and $42 \%$ yield, respectively). Therefore, we examined other conditions from Table 1 that had also delivered arylated product and found that a change of base to $\mathrm{NaO} t \mathrm{Bu}$, together with an increase in the temperature, delivered an arylation reaction that was more reliable across a wider range of ketones (for example, compounds 19 and 20 were now formed in 53 and $73 \%$ yield, respectively).

With these new arylation conditions, the one-pot synthesis of various carbolines was then possible in excellent yields. Using this approach, alkyl substituents could be incorporated with ease at either the C-3 or C-4 positions on the carboline nucleus (Table 3). Note that attempts at the one-pot synthesis of 18 via the dicarbonyl intermediate 17 were unsuccessful.

Table 3: One-pot synthesis of substituted $\beta$-Carbolines
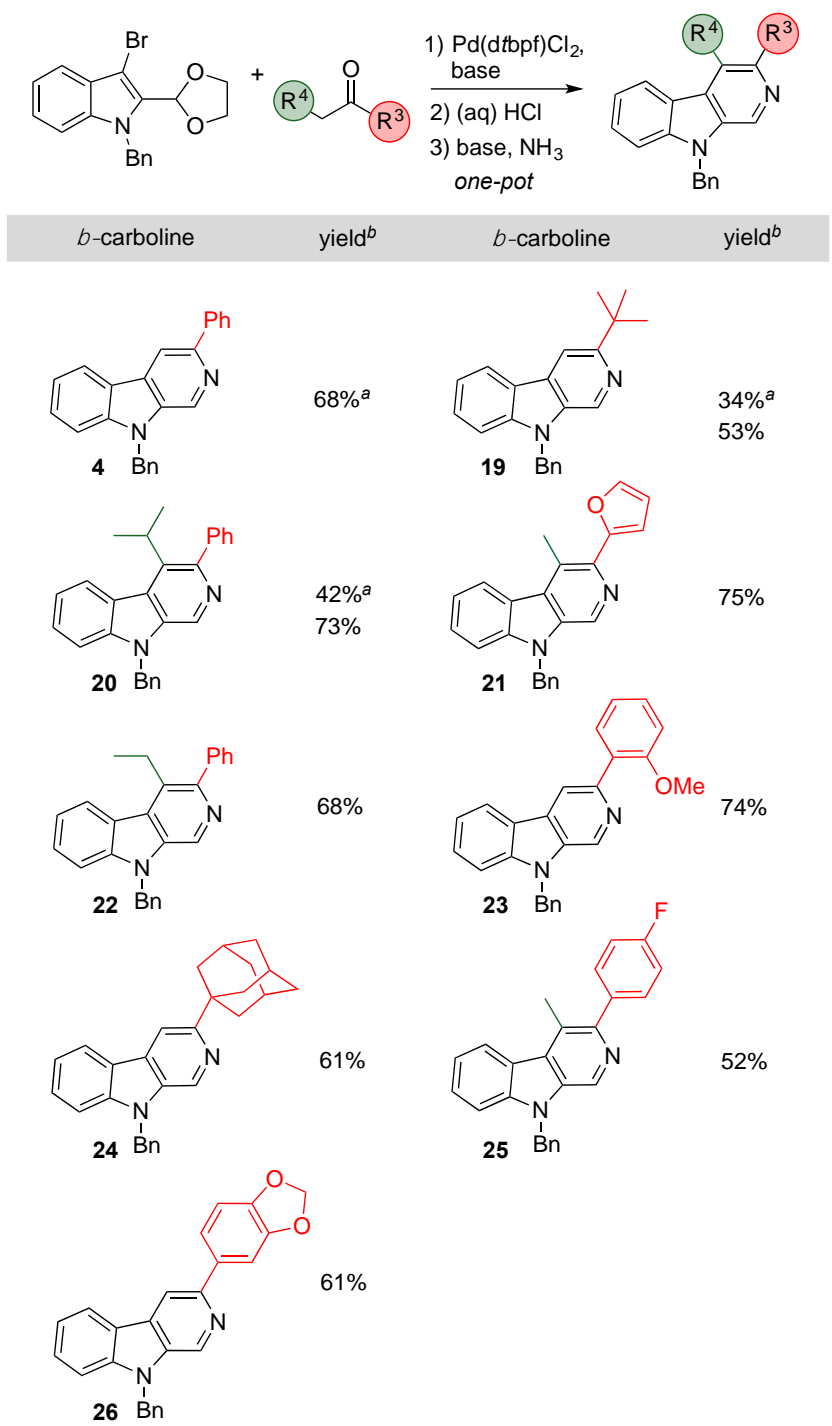

Reaction conditions: 1 ) Indole (1.0 equiv), $\mathrm{Pd}(\mathrm{dtbpf}) \mathrm{Cl}_{2}(5.0 \mathrm{~mol} \%)$, $\mathrm{NaOtBu}$ (2.5 equiv), ketone (2.0 equiv), THF, $75^{\circ} \mathrm{C}, 24 \mathrm{~h}$; 2) (aq.) $1 \mathrm{M} \mathrm{HCl}$; 3) $\mathrm{NaHCO}_{3}$ (20 equiv), $\mathrm{NH}_{3}$ (20 equiv, $7 \mathrm{M}$ in MeOH), EtOH, DMF, $100{ }^{\circ} \mathrm{C}, 12 \mathrm{~h}$. ${ }^{a}$ LiHMDS (2.5 eq.) as base, $50^{\circ} \mathrm{C}$

${ }^{b}$ Isolated yields

Next we examined the direct functionalization of the arylated products in situ, which was accomplished by quenching the arylation reaction of a methyl ketone with an electrophile (see $\mathbf{1} \rightarrow \mathbf{2 7}$ with the addition of benzyl bromide, Scheme 3). This protocol works well because the initial arylation reaction requires at least two equivalents of base to reach completion and, therefore, the initial arylation product is actually present in the reaction mixture as an enolate, which can be quenched. As expected, the benzylated compound $\mathbf{2 7}$ was then easily aromatized to carboline $\mathbf{2 8}$ under standard conditions (Scheme 3). 
Scheme 3: in situ enolate alkyation to furnish C-4 substituted $\beta$ carbolines

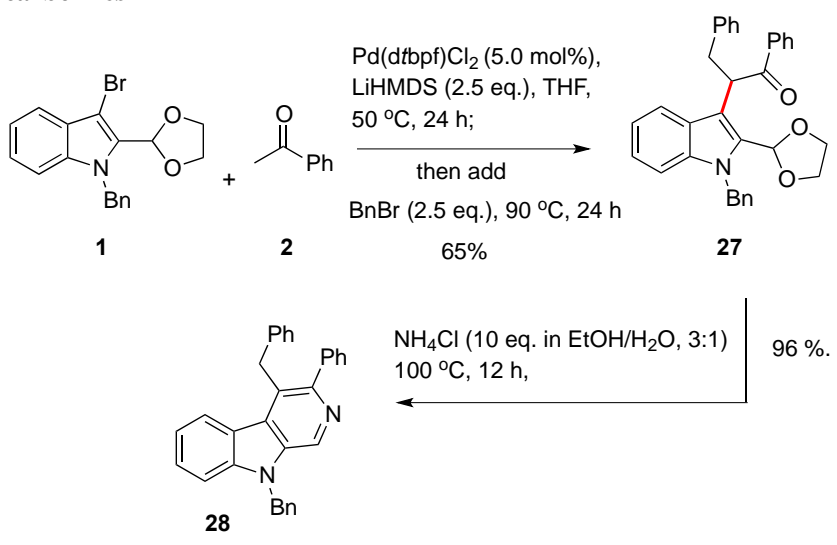

This precedent then provided the basis for a one-pot arylation/enolate-quench/aromatization sequence, starting from $\mathbf{1}$ and 2, that provided several C-4 derivatised products in good yields, (see 14, 28-31, Table 4). This chemistry represents a particularly convenient and short route to C-3,4disubstituted carbolines which installs the desired functionality with complete control of regiochemistry.

Table 4: One-pot synthesis of C-4 functionalized $\beta$-carbolines

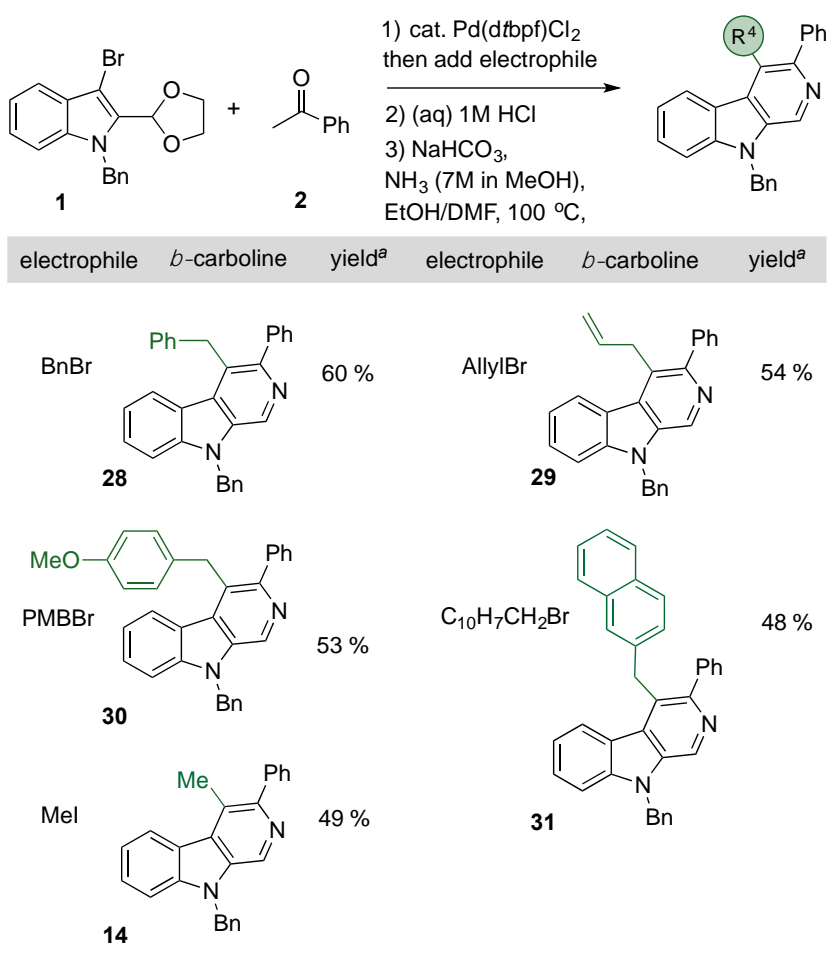

Reaction conditions: 1 ) Indole (1.0 equiv), $\mathrm{Pd}(\mathrm{dtbpf}) \mathrm{Cl}_{2}$ ( $\left.5.0 \mathrm{~mol} \%\right)$, LiHMDS (2.5 equiv), ketone (2.0 equiv), THF, $50^{\circ} \mathrm{C}, 24 \mathrm{~h}$; then electrophile, $90^{\circ} \mathrm{C}$; ${ }^{a}$ Isolated yields

To conclude, we have extended the enolate arylation/aromatization sequence to accomplish the synthesis of carbolines by using indole-based bromides as coupling partners. A variety of (heterocyclic) substitution patterns were compatible with this approach, either by arylation of a functionalized ketone or by a direct enolate arylation and electrophilic quenching sequence. The ability to combine an enolate arylation with a one-pot aromatization sequence is particularly advantageous. This convenient and modular approach allows access to a wide range of carboline derivatives with many potential uses.

\section{Experimental Section:}

General Methods: All reagents were used as purchased. Solvents were dried using standard laboratory techniques. All reactions requiring dry equipment were carried out in flamedried glassware under argon atmosphere. Flash column chromatography was performed using Geduran ${ }^{\circledR}$ silica gel 60 (40-63 $\mu \mathrm{m})$. Thin layer chromatography was performed on Merck Kieselgel 60 F254 0.25 mm pre-coated aluminumbacked plates. Product spots were visualized under UV light $(\lambda \max =254 \mathrm{~nm})$ and/or by staining with vanillin, phosphomolybdic acid or basic potassium permanganate solutions. ${ }^{1} \mathrm{H}$ nuclear magnetic resonance (NMR) spectra were recorded using a Bruker AVII400, AVIII400, or AVII500 instruments at $400 \mathrm{MHz}$ or $500 \mathrm{MHz} .{ }^{13} \mathrm{C}$ NMR spectra were recorded at $100 \mathrm{MHz}$ or $125 \mathrm{MHz} .{ }^{19} \mathrm{~F}$ NMR spectrum was recorded at $376 \mathrm{MHz}$. Chemical shifts, $\delta$, are reported relative to residual solvent peaks and quoted in parts per million (ppm) to the nearest 0.01 for ${ }^{1} \mathrm{H}$ and to the nearest $0.1 \mathrm{ppm}$ for ${ }^{13} \mathrm{C}$ and ${ }^{19} \mathrm{~F}$. Coupling constants, J, are quoted to the nearest 0.1 Hz. Assignments were based upon DEPT, COSY, HSQC, and HMBC experiments. High-resolution mass spectra were acquired using electrospray ionisation (ESI) as ionization source and were recorded on a Fisons Platform II with TOF detector. Infrared spectra (IR) were obtained from evaporated films using a Bruker Tensor 27 spectrometer, equipped with a PIKE Miracle Attenuated Total Reflectance (ATR) sampling accessory. Absorption maxima are quoted in wavenumbers $\left(\mathrm{cm}^{-1}\right)$ for the range $3500-600 \mathrm{~cm}^{-1}$. Melting points (m.p.) were obtained by using a Leica VMTG heated-stage microscope and are uncorrected.

General Procedure A for $\alpha$-Arylation of ketones. A fresh solution of LiHMDS was prepared in a dry vial adding sequentially dry THF $(2 \mathrm{~mL})$, HMDS $(80 \mu \mathrm{L}, 0.38 \mathrm{mmol}, 2.5$ eq.), a $2.5 \mathrm{M}$ solution of $n \mathrm{BuLi}(0.15 \mathrm{~mL}, 0.38 \mathrm{mmol} 2.5$ eq.) and stirring at $-78{ }^{\circ} \mathrm{C}$ for 10 minutes. The ketone $(0.31 \mathrm{mmol}$, 2.0 eq.) was then added at $0{ }^{\circ} \mathrm{C}$ and stirred for 15 minutes. In a second dry vial were added bromo indole $(0.15 \mathrm{mmol})$ and $\mathrm{Pd}(\mathrm{d} t \mathrm{bpf}) \mathrm{Cl}_{2}(5 \mathrm{mg}, 8 \mu \mathrm{mol}, 5 \mathrm{~mol} \%)$. The flask was sealed, evacuated and backfilled with argon. The freshly formed enolate solution was then transferred via syringe to the flask. The mixture was stirred at $50{ }^{\circ} \mathrm{C}$ for 24 hours in an oil bath. The resulting mixture was filtered through a plug of silica and concentrated in vacuo. The crude product was purified by flash column chromatography (eluted with a mixture of petroleum ether/ EtOAc) to afford the ketone product.

General Procedure B for the Cyclization of $\alpha$-Arylated Ketones. To a reaction flask were added the arylated ketone $(4.15 \mathrm{mmol})$ and a $1 \mathrm{M}$ solution of $\mathrm{NH}_{4} \mathrm{Cl}(4.1 \mathrm{~mL}, 10$ eq. $)$ in $\mathrm{EtOH} / \mathrm{H}_{2} \mathrm{O} 3: 1$. The mixture was stirred at $90{ }^{\circ} \mathrm{C}$ for $12 \mathrm{~h}$. 
$\mathrm{NH}_{4} \mathrm{HCO}_{3}(6.97 \mathrm{~g}, 83.0 \mathrm{mmol}, 20$ eq. $)$ was then added to the flask and the solution was stirred at $90{ }^{\circ} \mathrm{C}$ for 3 hours. The crude product was concentrated in vacuo, re-dissolved in pure EtOAc and mixed with water. The aqueous layer was extracted twice with EtOAc; the organic extracts were combined, dried over $\mathrm{MgSO}_{4}$, filtered and concentrated in vacuo. The resulting solid was purified by flash column chromatography (eluted with a mixture of petroleum ether/ EtOAc) to afford the $\beta$-carboline product.

General Procedure $C$ for the Synthesis of Ketones from Weinreb Amide 7. To a dry reaction flask were added indole $7(2.00 \mathrm{~g}, 6.79 \mathrm{mmol})$ and dry THF $(68 \mathrm{~mL})$. A 3M solution of the appropriate Grignard reagent $(6.8 \mathrm{~mL}, 20 \mathrm{mmol}, 3$ eq. $)$ was slowly added over 30 minutes at $-78{ }^{\circ} \mathrm{C}$ and then allowed to stir at $0{ }^{\circ} \mathrm{C}$ for 1 hour. The mixture was then quenched at 0 ${ }^{\circ} \mathrm{C}$ with $\mathrm{NH}_{4} \mathrm{Cl}_{\text {(aq.) }}$ and the aqueous layer was extracted twice with EtOAc. The organic extracts were combined, dried over $\mathrm{MgSO}_{4}$, filtered and concentrated in vacuo. The resulting solid was purified by flash column chromatography (eluted with a mixture of petroleum ether/ EtOAc) to afford the product ketone.

General Procedure D for the One-pot Synthesis of $\boldsymbol{\beta}$-Carbolines. To a dry vial were added bromo indole $\mathbf{1}$ (110 mg, $307 \mu \mathrm{mol}), \quad \mathrm{Pd}(\mathrm{d} t \mathrm{bpf}) \mathrm{Cl}_{2} \quad(10 \mathrm{mg}, 15 \mu \mathrm{mol}$, $5 \mathrm{~mol} \%$ ) and $\mathrm{NaO} t \mathrm{Bu}$ (74 mg, $0.77 \mathrm{mmol}, 2.5$ eq.). The flask was sealed, evacuated and backfilled with argon twice and then dry THF $(4 \mathrm{~mL})$ and the corresponding ketone $(0.61$ mmol, 2 eq.) were added in sequence. The mixture was stirred for 24 hours at $75{ }^{\circ} \mathrm{C}$. After cooling to room temperature, $\mathrm{HCl}_{\text {(aq.) }}(1 \mathrm{M}, 10$ eq.) was added and the mixture was stirred for 12 hours at $90{ }^{\circ} \mathrm{C}$. After cooling to room temperature, DMF (2 mL), EtOH (4 mL), $\mathrm{NaHCO}_{3}$ (516 mg, $6.14 \mathrm{mmol}, 20$ eq.,) and $\mathrm{NH}_{3}$ (7 $\mathrm{M}$ solution in $\mathrm{MeOH}, 0.88 \mathrm{~mL}, 6.1 \mathrm{mmol}, 20$ eq.) were added and the mixture was stirred at $110{ }^{\circ} \mathrm{C}$ for 24 hours. The crude product was concentrated in vacuo, redissolved in pure EtOAc and mixed with water. The aqueous layer was extracted twice with EtOAc and the organic extracts were combined, dried over $\mathrm{MgSO}_{4}$, filtered and concentrated in vacuo. The resulting solid was purified by flash column chromatography (eluted with a mixture of petroleum ether/ EtOAc) to afford the $\beta$-carboline product.

General Procedure $E$ for the Bromination of KetoIndoles. To a dry reaction flask were added the appropriate ketone $(5.45 \mathrm{mmol})$ and DMF $(3.9 \mathrm{~mL})$. A solution of NBS (1.11 g, $6.27 \mathrm{mmol}, 1.15$ eq.) in DMF (3.9 mL) was added over 30 minutes at $0{ }^{\circ} \mathrm{C}$ and the resulting mixture was then stirred at room temperature for 2 hours. $70 \mathrm{~mL}$ of $\mathrm{H}_{2} \mathrm{O}$ was added and the resulting slurry was extracted three times with EtOAc; the organic extracts were combined and washed five times with $\mathrm{H}_{2} \mathrm{O}$, dried over $\mathrm{MgSO}_{4}$, filtered and concentrated in vacuo, affording the bromo indole product or, alternatively, were purified by flash column chromatography (eluted with a mixture of petroleum ether/ EtOAc) to afford the bromo indole product.

General Procedure F for the One-pot Synthesis of $\boldsymbol{\beta}$-Carboline with addition of electrophiles. A fresh solution of LiHMDS was prepared in a dry vial adding sequentially dry THF (4 mL), HMDS (0.16 mL, $0.77 \mathrm{mmol}, 2.5$ eq.) and a 2.5 $\mathrm{M}$ solution of $n \mathrm{BuLi}(0.31 \mathrm{~mL}, 0.77 \mathrm{mmol}, 2.5$ eq.) and stirring at $-78{ }^{\circ} \mathrm{C}$ for 10 minutes. Acetophenone $(72 \mu \mathrm{L}, 0.61$ mmol, 2.0 eq.) was then added at $0{ }^{\circ} \mathrm{C}$ and stirred for 15 minutes. In a second dry vial were added bromo indole $\mathbf{1}(110$ $\mathrm{mg}, 307 \mu \mathrm{mol})$ and $\mathrm{Pd}(\mathrm{d} t \mathrm{bpf}) \mathrm{Cl}_{2}(10 \mathrm{mg}, 15 \mu \mathrm{mol}, 5 \mathrm{~mol} \%)$. The flask was sealed, evacuated and backfilled with argon. The freshly formed enolate solution was then transferred via syringe to the flask. The mixture was stirred at $50{ }^{\circ} \mathrm{C}$ for 24 hours in an oil bath. After cooling to room temperature, the appropriate electrophile ( $0.77 \mathrm{mmol}, 2.5$ eq. $)$ was added and the mixture was stirred at $90{ }^{\circ} \mathrm{C}$ for 24 hours. After cooling to room temperature, $1 \mathrm{M} \mathrm{HCl}_{\text {(aq.) }}(3.1 \mathrm{~mL}, 10$ eq. $)$ was added and the mixture was stirred for 12 hours at $90{ }^{\circ} \mathrm{C}$. After cooling to room temperature, DMF $(2 \mathrm{~mL}), \mathrm{EtOH}(4 \mathrm{~mL}), \mathrm{NaHCO}_{3}(516$ $\mathrm{mg}, 6.14$ mmol, 20 eq.,) and $\mathrm{NH}_{3}$ (7 $\mathrm{M}$ solution in $\mathrm{MeOH}$, $0.88 \mathrm{~mL}, 6.1 \mathrm{mmol}, 20$ eq.) were added and the mixture was stirred at $110{ }^{\circ} \mathrm{C}$ for 24 hours. The crude product was concentrated in vacuo, re-dissolved in pure EtOAc and mixed with water. The aqueous layer was extracted twice with EtOAc and the organic extracts were combined, dried over $\mathrm{MgSO}_{4}$, filtered and concentrated in vacuo. The resulting solids were purified by flash column chromatography (eluted with a mixture of petroleum ether/ EtOAc) to afford the $\beta$ carboline product.

Synthesis of 1-benzyl-3-bromo-2-(1,3-dioxolan-2-yl)-1Hindole (1). To a dry reaction flask connected to a Dean-Stark apparatus were added 1-benzyl-3-bromo- $1 \mathrm{H}$-indole-2carbaldehyde (330 mg, $1.05 \mathrm{mmol})$, ethylene glycol $(116 \mu \mathrm{L}$, 2.09 mmol, 2 eq.), $p$-toluenesulfonic acid monohydrate (20 $\mathrm{mg}, 105 \mu \mathrm{mol}, 10 \mathrm{~mol} \%)$ and toluene $(10 \mathrm{~mL})$. The resulting mixture was heated at reflux for 14 hours and then cooled to room temperature and quenched with $\mathrm{NaHCO}_{3 \text { (aq.). }}$. The aqueous layer was extracted twice with EtOAc, the organic extracts combined, dried over $\mathrm{MgSO}_{4}$, filtered and concentrated in vacuo. The resulting solid was purified by flash column chromatography (eluted with 9:1 petroleum ether/EtOAc), affording $\mathbf{1}(350 \mathrm{mg}, 93 \%)$ as a yellow solid. Mp: $64-67{ }^{\circ} \mathrm{C}$. IR $v_{\max }$ (thin film) 3060, 2888, $1075 \mathrm{~cm}^{-1}$. HRMS: calculated for $\mathrm{C}_{18} \mathrm{H}_{17} \mathrm{BrNO}_{2}, 358.04372[\mathrm{M}+\mathrm{H}]^{+}$, found $m / z$ 358.04379, $\Delta=0.20 \mathrm{ppm} .{ }^{1} \mathrm{H} \mathrm{NMR}(400 \mathrm{MHz}$, $\left.\mathrm{CDCl}_{3}\right) \delta_{\mathrm{H}}: 7.62-7.55\left(1 \mathrm{H}, \mathrm{m}, \mathrm{HC}_{\mathrm{Ar}}\right), 7.30-7.00(8 \mathrm{H}, \mathrm{m}, 8 \times$ $\left.H_{\mathrm{Ar}}\right), 6.21\left(1 \mathrm{H}, \mathrm{s}, \mathrm{CH}(\mathrm{OR})_{2}\right), 5.46\left(1 \mathrm{H}, \mathrm{s}, \mathrm{PhCH}_{2} \mathrm{R}\right), 4.06-$ $3.86\left(4 \mathrm{H}, \mathrm{m},\left(\mathrm{OCH}_{2}\right)_{2}\right) ;{ }^{13} \mathrm{C} \mathrm{NMR}\left(100 \mathrm{MHz}, \mathrm{CDCl}_{3}\right) \delta_{\mathrm{C}}$ : 137.9, 137.4, $129.3\left(3 \times C_{\mathrm{Ar}}\right), 128.6\left(\mathrm{H} C_{\mathrm{Ar}}\right), 127.3\left(\mathrm{H} C_{\mathrm{Ar}}\right)$, $126.7\left(\mathrm{C}_{\mathrm{Ar}}\right), 126.3,124.3,120.7,120.0,110.6\left(5 \times \mathrm{H} C_{\mathrm{Ar}}\right), 98.7$ $\left(\mathrm{CH}(\mathrm{OR})_{2}\right), 94.5(\mathrm{C}(3)), 65.2\left(\left(\mathrm{OCH}_{2}\right)_{2}\right), 48.5\left(\mathrm{PhCH}_{2} \mathrm{R}\right)$.

2-(1-Benzyl-2-(1,3-dioxolan-2-yl)-1H-indol-3-yl)-1-

phenylethan-1-one (3). This compound was prepared according to general procedure A, affording 3 (57 mg, 93\%) as a brown solid. Mp: $100-102{ }^{\circ} \mathrm{C}$. IR $v_{\max }$ (thin film) 3368 , 3060, 2925, 1613, 1082, $745 \mathrm{~cm}^{-1}$. HRMS: calculated for $\mathrm{C}_{26} \mathrm{H}_{24} \mathrm{O}_{3} \mathrm{~N}, 398.17507[\mathrm{M}+\mathrm{H}]^{+}$, found $m / z$ 398.17496, $\Delta=-$ $0.30 \mathrm{ppm} .{ }^{1} \mathrm{H}$ NMR $\left(400 \mathrm{MHz}, \mathrm{CDCl}_{3}\right) \delta_{\square}: 8.15-8.10(2 \mathrm{H}, \mathrm{m}$, $\left.2 \times H \mathrm{C}_{\mathrm{Ar}}\right), 7.63-7.54\left(2 \mathrm{H}, \mathrm{m}, 2 \times H \mathrm{C}_{\mathrm{Ar}}\right), 7.48(2 \mathrm{H}, \mathrm{t}, J=7.4$ $\left.\mathrm{Hz}, 2 \times H \mathrm{C}_{\mathrm{Ar}}\right), 7.33-7.03\left(9 \mathrm{H}, \mathrm{m}, 9 \times \mathrm{HC}_{\mathrm{Ar}}\right), 6.13(1 \mathrm{H}, \mathrm{s}$, $\left.\mathrm{CH}(\mathrm{OR})_{2}\right), 5.54\left(1 \mathrm{H}, \mathrm{s}, \mathrm{PhCH}_{2} \mathrm{R}\right), 4.65\left(2 \mathrm{H}, \mathrm{s}, \mathrm{CH}_{2} \mathrm{C}(\mathrm{O})\right)$, 4.05-3.90 (4H, m, $\left.\left(\mathrm{OCH}_{2}\right)_{2}\right) ;{ }^{13} \mathrm{C} \mathrm{NMR}\left(100 \mathrm{MHz}, \mathrm{CDCl}_{3}\right) \delta_{\mathrm{C}}$ : $196.7(\mathrm{C}(\mathrm{O})), 137.1,136.4,135.9\left(3 \times C_{\mathrm{Ar}}\right), 131.8\left(\mathrm{H}_{\mathrm{Ar}}\right)$, $129.8\left(C_{\mathrm{Ar}}\right), 127.5,127.5,127.4\left(3 \times \mathrm{H} C_{\mathrm{Ar}}\right), 126.6\left(C_{\mathrm{Ar}}\right)$, $126.0,125.0,121.9,118.7,118.2,109.0\left(6 \times \mathrm{HC}_{\mathrm{Ar}}\right), 108.2$ 
$\left(C_{\mathrm{Ar}}\right), 97.9\left(\mathrm{CH}(\mathrm{OR})_{2}\right), 63.9\left(\left(\mathrm{OCH}_{2}\right)_{2}\right), 46.7\left(\mathrm{RCH}_{2} \mathrm{Ph}\right), 34.0$ $\left(\mathrm{CH}_{2} \mathrm{C}(\mathrm{O})\right)$.

9-Benzyl-3-phenyl-9H-pyrido[3,4-b]indole (4). This compound was prepared according to general procedure B on a $4.15 \mathrm{mmol}$ scale, affording $4(1.30 \mathrm{~g}, 94 \%)$ as a light brown solid. Mp: 143 - $146{ }^{\circ} \mathrm{C}$. IR $v_{\max }$ (thin film) $3059,3030,1460$, 733, $694 \mathrm{~cm}^{-1}$. HRMS: calculated for $\mathrm{C}_{24} \mathrm{H}_{19} \mathrm{~N}_{2}, 335.15428$ $[\mathrm{M}+\mathrm{H}]^{+}$, found $m / z$ 335.15350, $\Delta=-2.3 \mathrm{ppm} .{ }^{1} \mathrm{H}$ NMR $(400$ $\left.\mathrm{MHz}, \mathrm{CDCl}_{3}\right) \delta_{\square}: 8.82(1 \mathrm{H}, \mathrm{s}, \mathrm{C}(1)), 8.33(1 \mathrm{H}, \mathrm{s}, \mathrm{C}(4)), 8.13$ $\left(1 \mathrm{H}, \mathrm{d}, J=7.8 \mathrm{~Hz}, H \mathrm{C}_{\mathrm{Ar}}\right), 8.08\left(2 \mathrm{H}, \mathrm{d}, J=7.5 \mathrm{~Hz}, H \mathrm{C}_{\mathrm{Ar}}\right)$, 7.55-7.43 $\left(3 \mathrm{H}, \mathrm{m}, 3 \times H \mathrm{C}_{\mathrm{Ar}}\right), 7.40-7.31\left(2 \mathrm{H}, \mathrm{m}, 2 \times H \mathrm{C}_{\mathrm{Ar}}\right)$, 7.30-7.15 $\left(4 \mathrm{H}, \mathrm{m}, 4 \times H \mathrm{C}_{\mathrm{Ar}}\right), 7.14-7.05\left(2 \mathrm{H}, \mathrm{m}, 2 \times H \mathrm{C}_{\mathrm{Ar}}\right)$, $5.41\left(2 \mathrm{H}, \mathrm{s}, \mathrm{PhCH} \mathrm{H}_{2} \mathrm{Ar}\right) ;{ }^{13} \mathrm{C} \mathrm{NMR}\left(100 \mathrm{MHz}, \mathrm{CDCl}_{3}\right) \delta_{\mathrm{C}}$ : $147.9,141.9,140.6,136.5,136.0\left(5 \times C_{\mathrm{Ar}}\right), 131.9(\mathrm{HC}(1))$, $129.8\left(C_{\mathrm{Ar}}\right), 129.0,128.8,128.6,127.9,127.8,126.9,126.7$, $122.0\left(8 \times \mathrm{H}_{\mathrm{Ar}}\right), 121.7\left(C_{\mathrm{Ar}}\right), 120.0,111.4,109.9\left(3 \times \mathrm{H} C_{\mathrm{Ar}}\right)$, $47.0\left(\mathrm{PhCH} \mathrm{H}_{2} \mathrm{Ar}\right) .{ }^{1} \mathrm{H}$ and ${ }^{13} \mathrm{C} \mathrm{NMR}$ data were consistent with those previously reported. ${ }^{7 \mathrm{c}}$

Synthesis of 3-Phenyl-9H-pyrido[3,4-b]indole (5). To a dry reaction flask were added freshly sublimed $\mathrm{AlCl}_{3}(287 \mathrm{mg}$, $2.15 \mathrm{mmol}, 6$ eq.) and toluene $(1.8 \mathrm{~mL})$. A solution of $\beta$ carboline $4(120 \mathrm{mg}, 359 \mu \mathrm{mol})$ in toluene $(1.8 \mathrm{~mL})$ was added at $0{ }^{\circ} \mathrm{C}$ over 10 minutes and stirred at room temperature for 2 hours. The resulting mixture was quenched with $\mathrm{NaHCO}_{3 \text { (aq.) }}$ and the aqueous layer extracted twice with EtOAc. The organic extracts were combined, dried over $\mathrm{MgSO}_{4}$, filtered and concentrated in vacuo. The resulting solids were purified by flash column chromatography (eluted with a 19:1 mixture of $\mathrm{CHCl}_{3} / \mathrm{MeOH}$ ) to afford the $\beta$ carboline product $5(71 \mathrm{mg}, 88 \%)$ as a white solid. Mp: 226 $229^{\circ} \mathrm{C}$. IR $v_{\max }$ (thin film) $3125,3018,2923,2755,1137,738$, $696 \mathrm{~cm}^{-1}$. HRMS: calculated for $\mathrm{C}_{17} \mathrm{H}_{13} \mathrm{~N}_{2}, 245.10732$ $[\mathrm{M}+\mathrm{H}]^{+}$, found $m / z, 245.10730, \Delta=-0.10 \mathrm{ppm} .{ }^{1} \mathrm{H}$ NMR $(400$ $\left.\mathrm{MHz} \mathrm{CDCl}_{3}\right) \delta_{\mathrm{H}}: 8.99\left(1 \mathrm{H}, \mathrm{s}, \mathrm{HC}_{\mathrm{Ar}}\right), 8.57(1 \mathrm{H}, \mathrm{bs}, \mathrm{NH}), 8.40$ $\left(1 \mathrm{H}, \mathrm{s}, H \mathrm{C}_{\mathrm{Ar}}\right), 8.20\left(1 \mathrm{H}, \mathrm{d}, J=7.8 \mathrm{~Hz}, H \mathrm{C}_{\mathrm{Ar}}\right), 8.12-8.07(2 \mathrm{H}$, $\left.\mathrm{m}, 2 \times H \mathrm{C}_{\mathrm{Ar}}\right), 7.59-7.46\left(4 \mathrm{H}, \mathrm{m}, 4 \times H \mathrm{C}_{\mathrm{Ar}}\right), 7.43-7.36(1 \mathrm{H}, \mathrm{m}$, $\left.H \mathrm{C}_{\mathrm{Ar}}\right), 7.32\left(1 \mathrm{H}, \mathrm{dd}, J=8.1,7.0 \mathrm{~Hz}, H \mathrm{C}_{\mathrm{Ar}}\right) .{ }^{1} \mathrm{H} \mathrm{NMR}(400$ $\left.\mathrm{MHz}, \mathrm{DMSO}-\mathrm{d}_{6}\right) \delta_{\square}: 11.69(1 \mathrm{H}, \mathrm{s}, \mathrm{NH}), 9.00(1 \mathrm{H}, \mathrm{s}, H \mathrm{C}(1))$, $8.77(1 \mathrm{H}, \mathrm{s}, H \mathrm{C}(4)), 8.36\left(1 \mathrm{H}, \mathrm{d}, J=7.9 \mathrm{~Hz}, H \mathrm{C}_{\mathrm{Ar}}\right), 8.22(2 \mathrm{H}$, ddd, $\left.J=8.3,1.2,1.0 \mathrm{~Hz}, 2 \times H \mathrm{C}_{\mathrm{Ar}}\right), 7.66-7.60\left(1 \mathrm{H}, \mathrm{m}, H \mathrm{C}_{\mathrm{Ar}}\right)$, $7.58\left(1 \mathrm{H}, \mathrm{dd}, J=6.9,1.2 \mathrm{~Hz}, H \mathrm{C}_{\mathrm{Ar}}\right), 7.54-7.48(2 \mathrm{H}, \mathrm{m}, 2 \times$ $\left.H \mathrm{C}_{\mathrm{Ar}}\right), 7.41-7.35\left(1 \mathrm{H}, \mathrm{m}, H \mathrm{C}_{\mathrm{Ar}}\right), 7.28(1 \mathrm{H}, \mathrm{ddd}, J=8.0,6.9$, $\left.1.0 \mathrm{~Hz}, H_{\mathrm{Ar}}\right) ;{ }^{13} \mathrm{C}$ NMR $\left(100 \mathrm{MHz}, \mathrm{CDCl}_{3}\right) ;{ }^{13} \mathrm{C}$ NMR $(100$ $\left.\mathrm{MHz}, \mathrm{DMSO}-\mathrm{d}_{6}\right) \delta_{\mathrm{C}}: 146.0,141.6,140.6,135.9\left(4 \times C_{\mathrm{Ar}}\right)$, $134.0(\mathrm{HC}(1)), 129.3\left(C_{\mathrm{Ar}}\right), 129.1,128.7,127.9,126.6122 .5$, $\left(5 \times \mathrm{H}_{\mathrm{Ar}}\right), 121.5\left(C_{\mathrm{Ar}}\right), 119.8,112.5\left(2 \times \mathrm{H} C_{\mathrm{Ar}}\right), 111.6$ (HC(4)). ${ }^{1} \mathrm{H}$ NMR data were consistent with those previously reported. ${ }^{24}$

Synthesis of 1-Benzyl- $N$-methoxy- $N$-methyl-1H-indole-2carboxamide (7). To a dry reaction flask equipped with a reflux condenser were added indole $6(12.0 \mathrm{~g}, 58.7 \mathrm{mmol})$ and dry THF $(590 \mathrm{~mL})$. NaH $(60 \%$ in mineral oil, $2.82 \mathrm{~g}, 70.5$ mmol, 1.2 eq.) was added slowly at $0{ }^{\circ} \mathrm{C}$ over 10 minutes and the resulting mixture was heated at reflux for 30 minutes. After cooling to room temperature, benzyl bromide $(8.4 \mathrm{~mL}$, $70 \mathrm{mmol}, 1.2$ eq.) was added and the solution was heated at reflux for 2 hours and then cooled to $0{ }^{\circ} \mathrm{C}$, when it was quenched using $\mathrm{NH}_{4} \mathrm{Cl}_{(\mathrm{aq.} .)}$. The aqueous layer was extracted twice with EtOAc and the organic extracts were combined, dried over $\mathrm{MgSO}_{4}$, filtered and concentrated in vacuo. The resulting solid was purified by flash column chromatography (eluted with 4:1 petroleum ether/EtOAc) to afford protected indole $7(17.1 \mathrm{~g}, 99 \%)$ as a white solid. Mp: $34-37{ }^{\circ} \mathrm{C}$. IR $v_{\max }$ (thin film) 3060, 3030, 2931, 1632, 1453, $739 \mathrm{~cm}^{-1}$. HRMS: calculated for $\mathrm{C}_{18} \mathrm{H}_{18} \mathrm{O}_{2} \mathrm{~N}_{2}, 295.14410[\mathrm{M}+\mathrm{H}]^{+}$, found $m / z$ 295.14398, $\Delta=-0.41 \mathrm{ppm} .{ }^{1} \mathrm{H}$ NMR (400 $\left.\mathrm{MHz}, \mathrm{CDCl}_{3}\right) \delta$ : 7.79-7.40 $\left(1 \mathrm{H}, \mathrm{m}, H \mathrm{C}_{\mathrm{Ar}}\right), 7.44(1 \mathrm{H}, \mathrm{dd}, J=8.4,0.8 \mathrm{~Hz}$, $\left.H \mathrm{C}_{\mathrm{Ar}}\right), 7.33\left(1 \mathrm{H}, \mathrm{ddd}, J=8.3,7.0,1.2 \mathrm{~Hz}, H \mathrm{C}_{\mathrm{Ar}}\right), 7.31-7.19$ $\left(5 \mathrm{H}, \mathrm{m}, 6 \times \mathrm{HC}_{\mathrm{Ar}}\right), 7.13\left(2 \mathrm{H}, \mathrm{d}, J=6.6 \mathrm{~Hz}, 2 \times H_{\mathrm{Ar}}\right), 5.77$ $\left(2 \mathrm{H}, \mathrm{s}, \mathrm{PhCH}_{2} \mathrm{R}\right), 3.53\left(3 \mathrm{H}, \mathrm{s}, \mathrm{OCH}_{3}\right), 3.34\left(3 \mathrm{H}, \mathrm{s}, \mathrm{NCH}_{3}\right) ;{ }^{13} \mathrm{C}$ NMR $\left(100 \mathrm{MHz}, \mathrm{CDCl}_{3}\right) \delta_{\mathrm{C}}: 162.8(\mathrm{C}(\mathrm{O})), 138.6,138.4$, $129.6\left(3 \times C_{\mathrm{Ar}}\right), 128.6,127.3,126.8\left(3 \times \mathrm{H} C_{\mathrm{Ar}}\right), 126.7\left(C_{\mathrm{Ar}}\right)$, $124.4,122.3,120.6,110.6,108.1\left(5 \times \mathrm{HC}_{\mathrm{Ar}}\right), 61.2\left(\mathrm{OCH}_{3}\right)$, $47.9\left(\mathrm{RCH}_{2} \mathrm{Ph}\right), 33.9\left(\mathrm{NCH}_{3}\right)$.

1-(1-Benzyl-1H-indol-2-yl)ethan-1-one (8). This compound was prepared according to general procedure $\mathrm{C}$, affording $\mathbf{8}$ $(1.28 \mathrm{~g}, 76 \%)$ as a white solid. Mp: $125-126^{\circ} \mathrm{C}$. IR $v_{\max }$ (thin film) 3061, 3031, 2924, 1657, $725 \mathrm{~cm}^{-1}$. HRMS: calculated for $\mathrm{C}_{17} \mathrm{H}_{16} \mathrm{ON}, 250.12264[\mathrm{M}+\mathrm{H}]^{+}$, found $\mathrm{m} / z .250 .12302, \Delta=$ 1.50 ppm. ${ }^{1} \mathrm{H}$ NMR $\left(400 \mathrm{MHz}, \mathrm{CDCl}_{3}\right) \delta_{\square}: 7.81-7.77(1 \mathrm{H}, \mathrm{m}$, $\left.H \mathrm{C}_{\mathrm{Ar}}\right), 7.45-7.36\left(3 \mathrm{H}, \mathrm{m}, 3 \times \mathrm{HC}_{\mathrm{Ar}}\right), 7.32-7.20(4 \mathrm{H}, \mathrm{m}, 4 \times$ $\left.H \mathrm{C}_{\mathrm{Ar}}\right), 7.14-7.08\left(2 \mathrm{H}, \mathrm{m}, 2 \times \mathrm{HC}_{\mathrm{Ar}}\right), 5.91\left(\mathrm{~s}, 2 \mathrm{H}, \mathrm{NCH}_{2} \mathrm{Ph}\right)$, $2.66\left(\mathrm{~s}, 3 \mathrm{H}, \mathrm{C}(\mathrm{O}) \mathrm{CH}_{3}\right) ;{ }^{13} \mathrm{C} \mathrm{NMR}\left(100 \mathrm{MHz}, \mathrm{CDCl}_{3}\right) \delta_{\mathrm{C}}$ : $191.3(C(\mathrm{O})), 140.0,138.4,134.4\left(3 \times C_{\mathrm{Ar}}\right), 128.5,127.1$, $126.5,126.3\left(4 \times H C_{\mathrm{Ar}}\right), 126.0\left(C_{\mathrm{Ar}}\right), 123.0,121.1,113.0$, $111.0\left(4 \times \mathrm{HC}_{\mathrm{Ar}}\right), 48.2\left(\mathrm{PhCH}_{2} \mathrm{~N}\right), 28.1\left(\mathrm{C}(\mathrm{O}) \mathrm{CH}_{3}\right) .{ }^{1} \mathrm{H} \mathrm{NMR}$ data were consistent with those previously reported. ${ }^{25}$

(1-Benzyl-1H-indol-2-yl)(phenyl)methanone (9). This compound was prepared according to general procedure $\mathrm{C}$ on a $10.2 \mathrm{mmol}$ scale, affording $9(2.51 \mathrm{~g}, 79 \%)$ as a white solid. Mp: $107-110^{\circ} \mathrm{C}$. IR $v_{\max }$ (thin film) $3060,3030,2923,1633$, $718,694 \mathrm{~cm}^{-1}$. HRMS: calculated for $\mathrm{C}_{22} \mathrm{H}_{17} \mathrm{ONNa}, 334.12024$ $[\mathrm{M}+\mathrm{Na}]^{+}$, found $m / z, 334.12023, \Delta=-0.02 \mathrm{ppm} .{ }^{1} \mathrm{H}$ NMR $(400$ $\left.\mathrm{MHz}, \mathrm{CDCl}_{3}\right) \delta_{\square}: 7.95-7.90\left(2 \mathrm{H}, \mathrm{m}, 2 \times \mathrm{HC}_{\mathrm{Ar}}\right), 7.73(1 \mathrm{H}, \mathrm{d}, J$ $\left.=8.0, H \mathrm{C}_{\mathrm{Ar}}\right), 7.65-7.58\left(1 \mathrm{H}, \mathrm{m}, H \mathrm{C}_{\mathrm{Ar}}\right), 7.54-7.48(2 \mathrm{H}, \mathrm{m}, 2 \times$ $\left.H \mathrm{C}_{\mathrm{Ar}}\right), 7.44\left(1 \mathrm{H}, \mathrm{dd}, J=8.5,0.7 \mathrm{~Hz}, H \mathrm{C}_{\mathrm{Ar}}\right), 7.38(1 \mathrm{H}, \mathrm{ddd}, J=$ 8.4, 6.9, $\left.1.1 \mathrm{~Hz}, H \mathrm{C}_{\mathrm{Ar}}\right), 7.31-7.18\left(4 \mathrm{H}, \mathrm{m}, 4 \times H \mathrm{C}_{\mathrm{Ar}}\right), 7.18-$ $7.14\left(2 \mathrm{H}, \mathrm{m}, 2 \times H \mathrm{C}_{\mathrm{Ar}}\right), 7.12(1 \mathrm{H}, \mathrm{d}, J=0.6 \mathrm{~Hz}, \mathrm{C}(3)), 5.91$ $\left(2 \mathrm{H}, \mathrm{s}, \mathrm{PhCH}_{2} \mathrm{R}\right) ;{ }^{13} \mathrm{C} \mathrm{NMR}\left(100 \mathrm{MHz}, \mathrm{CDCl}_{3}\right) \delta_{\mathrm{C}}: 188.6$ $(C(\mathrm{O})), 140.2,139.4,138.4,134.7\left(4 \times C_{\mathrm{Ar}}\right), 132.2,129.8$, 128.6, 128.2, 127.2, 126.6, $126.2\left(7 \times \mathrm{H} C_{\mathrm{Ar}}\right), 126.1\left(C_{\mathrm{Ar}}\right)$, 123.1,121.1,115.8, $111.1\left(4 \times \mathrm{HC}_{\mathrm{Ar}}\right), 48.1\left(\mathrm{PhCH}_{2} \mathrm{R}\right) .{ }^{1} \mathrm{H} \mathrm{NMR}$ data were consistent with those previously reported. ${ }^{26}$

1-(1-Benzyl-3-bromo-1H-indol-2-yl)ethan-1-one (10). This compound was prepared according to general procedure $\mathrm{E}$, affording $10(1.66 \mathrm{~g}, 93 \%)$ as a white solid. Mp: $118-120^{\circ} \mathrm{C}$. IR $v_{\max }$ (thin film) 3061, 3032, 2921, 16511, $726 \mathrm{~cm}^{-1}$. HRMS: calculated for $\mathrm{C}_{17} \mathrm{H}_{15} \mathrm{ONBr}$, $328.03315[\mathrm{M}+\mathrm{H}]^{+}$, found $\mathrm{m} / \mathrm{z}$ 328.03348, $\Delta=0.99 \mathrm{ppm} .{ }^{1} \mathrm{H}$ NMR $\left(400 \mathrm{MHz}, \mathrm{CDCl}_{3}\right) \delta_{\square}$ : 7.80-7.74 $\left(1 \mathrm{H}, \mathrm{m}, H \mathrm{C}_{\mathrm{Ar}}\right), 7.43-7.39\left(2 \mathrm{H}, \mathrm{m}, 2 \times H \mathrm{C}_{\mathrm{Ar}}\right), 7.32-$ $7.22\left(4 \mathrm{H}, \mathrm{m}, 4 \times H \mathrm{C}_{\mathrm{Ar}}\right), 7.08-7.03\left(2 \mathrm{H}, \mathrm{m}, 2 \times H \mathrm{C}_{\mathrm{Ar}}\right), 5.80$ $\left(2 \mathrm{H}, \mathrm{s}, \mathrm{PhCH}_{2} \mathrm{~N}\right), 2.83\left(3 \mathrm{H}, \mathrm{s}, \mathrm{C}(\mathrm{O}) \mathrm{CH}_{3}\right) ;{ }^{13} \mathrm{C}$ NMR $(100$ $\left.\mathrm{MHz}, \mathrm{CDCl}_{3}\right) \delta_{\mathrm{C}}: 192.0(C(\mathrm{O})), 138.4,138.0,132.6\left(3 \times C_{\mathrm{Ar}}\right)$, $128.6,127.3,127.1\left(3 \times \mathrm{H} C_{\mathrm{Ar}}\right), 126.8\left(C_{\mathrm{Ar}}\right), 126.3,121.9$, 121.8, $111.0\left(4 \times \mathrm{HC}_{\mathrm{Ar}}\right), 100.0\left(C_{\mathrm{Ar}}\right), 48.9\left(\mathrm{PhCH}_{2} \mathrm{~N}\right), 31.9$ $\left(\mathrm{C}(\mathrm{O}) \mathrm{CH}_{3}\right)$.

(1-Benzyl-3-bromo-1H-indol-2-yl)(phenyl)methanone (11). This compound was prepared according to general procedure 
E on a $4.17 \mathrm{mmol}$ scale, affording $11(1.62 \mathrm{~g}, 99 \%)$ as a white solid. Mp: $87-89{ }^{\circ} \mathrm{C}$. IR $v_{\max }$ (thin film) 3060, 3030, 1719, 722, $692 \mathrm{~cm}^{-1}$. HRMS: calculated for $\mathrm{C}_{22} \mathrm{H}_{17} \mathrm{ONBr}, 390.04880$ $[\mathrm{M}+\mathrm{H}]^{+}$, found $m / z, 390.04959, \Delta=2.02 \mathrm{ppm} .{ }^{1} \mathrm{H}$ NMR $(400$ $\left.\mathrm{MHz}, \mathrm{CDCl}_{3}\right) \delta_{\square}: 7.82-7.78\left(2 \mathrm{H}, \mathrm{m}, 2 \times H \mathrm{C}_{\mathrm{Ar}}\right), 7.68(1 \mathrm{H}, \mathrm{d}, J$ $\left.=8.0 \mathrm{~Hz}, H \mathrm{C}_{\mathrm{Ar}}\right), 7.62-7.56\left(1 \mathrm{H}, \mathrm{m}, H \mathrm{C}_{\mathrm{Ar}}\right), 7.46-7.34(4 \mathrm{H}, \mathrm{m}, 4$ $\left.\times H \mathrm{C}_{\mathrm{Ar}}\right), 7.27\left(1 \mathrm{H}, \mathrm{ddd}, J=8.0,6.6,1.3 \mathrm{~Hz}, H \mathrm{C}_{\mathrm{Ar}}\right), 7.24-7.14$ $\left(3 \mathrm{H}, \mathrm{m}, 3 \times H_{\mathrm{Ar}}\right), 7.08-7.03\left(2 \mathrm{H}, \mathrm{m}, H \mathrm{C}_{\mathrm{Ar}}\right), 5.60(2 \mathrm{H}, \mathrm{s}$, $\left.\mathrm{PhCH}_{2} \mathrm{R}\right) ;{ }^{13} \mathrm{C}$ NMR $\left(100 \mathrm{MHz}, \mathrm{CDCl}_{3}\right) \delta_{\mathrm{C}}: 189.4(\mathrm{C}(\mathrm{O}))$, 138.0, 138.0, 137.5, $133.5\left(4 \times C_{\mathrm{Ar}}\right), 133.3,130.2,128.7$, $128.5,127.6\left(5 \times \mathrm{HC}_{\mathrm{Ar}}\right), 126.8\left(\mathrm{C}_{\mathrm{Ar}}\right), 126.7,126.2,121.6$, 121.3, $110.9\left(5 \times \mathrm{HC}_{\mathrm{Ar}}\right), 98.1\left(C_{\mathrm{Ar}}\right), 48.3\left(\mathrm{PhCH}_{2} \mathrm{R}\right)$.

Synthesis of 1-Benzyl-3-bromo-2-(2-methyl-1,3-dioxolan2-yl)-1H-indole (12). To a dry reaction flask connected to a Dean-Stark apparatus were added bromo indole 10 (3.00 g, $9.14 \mathrm{mmol})$, ethylene glycol (5.1 mL, $91 \mathrm{mmol}, 10$ eq.), $p$ toluenesulfonic acid monohydrate $(175 \mathrm{mg}, 914 \mu \mathrm{mol}, 10$ mol\%) and toluene $(91 \mathrm{~mL})$. The resulting mixture was heated at $120{ }^{\circ} \mathrm{C}$ for 60 hours and then cooled to room temperature and quenched with $\mathrm{NaHCO}_{3 \text { (aq.) }}$. The aqueous layer was extracted twice with EtOAc, the organic extracts combined, dried over $\mathrm{MgSO}_{4}$, filtered and concentrated in vacuo. The resulting solid was purified by flash column chromatography (eluted with 1:0.8 petroleum ether/ $\left.\mathrm{CHCl}_{3}\right)$, affording $\mathbf{1 2}(2.14$ $\mathrm{g}, 63 \%$ ) as a white solid. Mp: $89-90{ }^{\circ} \mathrm{C}$. IR $v_{\max }$ (thin film) 3031, 2990, $2892 \mathrm{~cm}^{-1}$. HRMS: calculated for $\mathrm{C}_{19} \mathrm{H}_{18} \mathrm{O}_{2} \mathrm{NBr}$, $372.05937[\mathrm{M}+\mathrm{H}]^{+}$, found $\mathrm{m} / z$ 372.05930, $\Delta=-0.19 \mathrm{ppm} .{ }^{1} \mathrm{H}$ NMR (400 MHz, $\left.\mathrm{CDCl}_{3}\right) \delta_{\square}: 7.59-7.53\left(1 \mathrm{H}, \mathrm{m}, H \mathrm{C}_{\mathrm{Ar}}\right), 7.20-$ $7.05\left(6 \mathrm{H}, \mathrm{m}, 6 \times H \mathrm{C}_{\mathrm{Ar}}\right), 7.85-6.79\left(2 \mathrm{H}, \mathrm{m}, 2 \times H \mathrm{C}_{\mathrm{Ar}}\right), 5.60$ $\left(2 \mathrm{H}, \mathrm{s}, \mathrm{PhCH}_{2} \mathrm{~N}\right), 3.95-3.83\left(2 \mathrm{H}, \mathrm{m} \mathrm{CH} \mathrm{H}_{\mathrm{b}} \mathrm{CH}_{a} \mathrm{H}_{\mathrm{b}}\right), 3.65-3.53$ $\left(2 \mathrm{H}, \mathrm{m}, \mathrm{CH}_{\mathrm{a}} \mathrm{H}_{\mathrm{b}} \mathrm{CH}_{\mathrm{a}} H_{\mathrm{b}}\right) ; 1.60\left(3 \mathrm{H}, \mathrm{s}, \mathrm{CCH}_{3}\right) ;{ }^{13} \mathrm{C} \mathrm{NMR}(100$ $\left.\mathrm{MHz}, \mathrm{CDCl}_{3}\right) \delta_{\mathrm{C}}: 138.4,137.1,135.4\left(3 \times C_{\mathrm{Ar}}\right), 128.6\left(\mathrm{H} C_{\mathrm{Ar}}\right)$, $127.4\left(C_{\mathrm{Ar}}\right), 127.0,125.7,123.6,120.7,119.8,110.3(6 \times$ $\left.\left.\mathrm{H} C_{\mathrm{Ar}}\right), 106.4,89.6\left(2 \times C_{\mathrm{Ar}}\right), 64.6\left(\mathrm{OCH}_{2}\right)_{2}\right), 48.6\left(\mathrm{PhCH}_{2} \mathrm{~N}\right)$, $26.4\left(\mathrm{CCH}_{3}\right)$.

2-(1-Benzyl-2-(1,3-dioxolan-2-yl)-1H-indol-3-yl)-1-

phenylpropan-1-one (13). This compound was prepared according to general procedure A, affording 13 (47 mg, 74\%) as a yellow solid. Mp: $159-164{ }^{\circ} \mathrm{C}$. IR $v_{\max }$ (thin film) 3061 , 2974, 2930, 2890, 1680, 1079, $745 \mathrm{~cm}^{-1}$. HRMS: calculated for $\mathrm{C}_{27} \mathrm{H}_{25} \mathrm{O}_{3} \mathrm{~N}, 412.19072[\mathrm{M}+\mathrm{H}]^{+}$, found $m / z$ 412.19196, $\Delta=$ 3.0 ppm. ${ }^{1} \mathrm{H}$ NMR $\left(400 \mathrm{MHz}, \mathrm{CDCl}_{3}\right) \delta_{\square}: 8.01(2 \mathrm{H}, \mathrm{d}, J=$ $\left.7.40 \mathrm{~Hz}, 2 \times H \mathrm{C}_{\mathrm{Ar}}\right), 7.64\left(1 \mathrm{H}, \mathrm{d}, J=7.6 \mathrm{~Hz}, H \mathrm{C}_{\mathrm{Ar}}\right), 7.34(1 \mathrm{H}$, $\left.\mathrm{t}, J=7.4 \mathrm{~Hz}, H \mathrm{C}_{\mathrm{Ar}}\right), 7.24\left(2 \mathrm{H}, \mathrm{t}, J=7.4 \mathrm{~Hz}, 2 \times H \mathrm{C}_{\mathrm{Ar}}\right), 7.21-$ $7.13\left(3 \mathrm{H}, \mathrm{m}, 3 \times \mathrm{HC}_{\mathrm{Ar}}\right), 7.08-6.99\left(3 \mathrm{H}, \mathrm{m}, 3 \times \mathrm{HC}_{\mathrm{Ar}}\right), 6.90-$ $6.84\left(2 \mathrm{H}, \mathrm{m}, 2 \times \mathrm{HC}_{\mathrm{Ar}}\right), 6.18\left(1 \mathrm{H}, \mathrm{s}, \mathrm{CH}(\mathrm{OR})_{2}\right), 5.42(1 \mathrm{H}, \mathrm{s}$, $\left.\mathrm{PhCH}_{a} \mathrm{H}_{\mathrm{b}} \mathrm{R}\right), 5.41\left(1 \mathrm{H}, \mathrm{s}, \mathrm{PhCH}_{\mathrm{a}} H_{b} \mathrm{R}\right), 5.15(1 \mathrm{H}, \mathrm{q}, J=6.8$, $\left.\mathrm{CHCH}_{3}\right), 4.12-3.92\left(4 \mathrm{H}, \mathrm{m},\left(\mathrm{OCH}_{2}\right)_{2}\right), 1.66\left(3 \mathrm{H}, \mathrm{d}, \mathrm{CH}_{3} \mathrm{CH}\right)$; ${ }^{13} \mathrm{C} \mathrm{NMR}\left(100 \mathrm{MHz}, \mathrm{CDCl}_{3}\right) \delta_{\mathrm{C}}: 201.1(\mathrm{C}(\mathrm{O})), 138.1,137.64$, $137.0(3 \times C \mathrm{Ar}), 132.4,128.8\left(2 \times \mathrm{H}_{\mathrm{Ar}}\right), 128.7\left(C_{\mathrm{Ar}}\right), 128.6$, 128.2, 127.1, $125.9\left(4 \times \mathrm{H} C_{\mathrm{Ar}}\right), 125.8\left(C_{\mathrm{Ar}}\right), 123.1,120.2$, $119.9\left(3 \times \mathrm{HC}_{\mathrm{Ar}}\right), 116.7\left(C_{\mathrm{Ar}}\right), 110.1\left(\mathrm{H} C_{\mathrm{Ar}}\right), 98.6\left(C \mathrm{H}\left(\mathrm{OR}_{2}\right)_{2}\right)$, $65.2 \quad\left(\mathrm{CHO}_{2} \mathrm{C}_{a} \mathrm{H}_{2} \mathrm{C}_{\mathrm{b}} \mathrm{H}_{2}\right), \quad 65.0 \quad\left(\mathrm{CHO}_{2} \mathrm{C}_{a} \mathrm{H}_{2} \mathrm{C}_{\mathrm{b}} \mathrm{H}_{2}\right), \quad 47.7$ $\left(\mathrm{PhCH}_{2} \mathrm{R}\right), 39.8\left(\mathrm{CHCH}_{3}\right), 18.0\left(\mathrm{CHCH}_{3}\right)$.

9-Benzyl-4-methyl-3-phenyl-9H-pyrido[3,4-b]indole (14). This compound was prepared according to general procedure B on a $0.15 \mathrm{mmol}$ scale, affording 14 (50 mg, 94\%) as a yellow solid. Mp: $164-167{ }^{\circ} \mathrm{C}$. IR $v_{\max }$ (thin film) 3055 , 3030, 2924, 1454, 737, $701 \mathrm{~cm}^{-1}$. HRMS: calculated for
$\mathrm{C}_{25} \mathrm{H}_{21} \mathrm{~N}_{2}, 349.16993[\mathrm{M}+\mathrm{H}]^{+}$, found $m / z, 349.16946, \Delta=-1.3$ ppm. ${ }^{1} \mathrm{H}$ NMR $\left(400 \mathrm{MHz}, \mathrm{CDCl}_{3}\right) \delta_{\square}: 8.82(1 \mathrm{H}, \mathrm{s}, H \mathrm{C}(1))$, $8.33\left(1 \mathrm{H}, \mathrm{d}, J=8.0 \mathrm{~Hz}, H \mathrm{C}_{\mathrm{Ar}}\right), 7.65-7.60\left(2 \mathrm{H}, \mathrm{m}, 2 \times H \mathrm{C}_{\mathrm{A}}\right)$, $7.59\left(1 \mathrm{H}\right.$, ddd $\left.J=8.3,7.1,1.2 \mathrm{~Hz}, H \mathrm{C}_{\mathrm{A}}\right), 7.54-7.47(3 \mathrm{H}, \mathrm{m}, 3$ $\left.\times H \mathrm{C}_{\mathrm{A}}\right), 7.45-7.39\left(1 \mathrm{H}, \mathrm{m}, H \mathrm{C}_{\mathrm{Ar}}\right) 7.34(1 \mathrm{H}, \mathrm{t}, J=8.0 \mathrm{~Hz}$, $H C(6)), 7.32-7.25\left(3 \mathrm{H}, \mathrm{m}, 3 \times \mathrm{C}_{\mathrm{Ar}}\right), 7.22-7.17(2 \mathrm{H}, \mathrm{m}, 2 \times$ $\left.\mathrm{HC}_{\mathrm{Ar}}\right), 5.58\left(2 \mathrm{H}, \mathrm{s}, \mathrm{PhCH}_{2} \mathrm{R}\right), 2.91\left(3 \mathrm{H}, \mathrm{s}, \mathrm{RCH}_{3}\right) ;{ }^{13} \mathrm{C} \mathrm{NMR}$ $\left(100 \mathrm{MHz}, \mathrm{CDCl}_{3}\right) \delta_{\mathrm{C}}: 149.2,141.7,141.2,136.6,135.6(5 \times$ $\left.\mathrm{C}_{\mathrm{Ar}}\right), 130.0,129.3\left(2 \times \mathrm{HC}_{\mathrm{Ar}}\right), 129.0(C(1)), 128.4\left(\mathrm{C}_{\mathrm{Ar}}\right)$, $128.1,127.8,127.8,127.2,126.6\left(5 \times \mathrm{H} C_{\mathrm{Ar}}\right), 124.9\left(C_{\mathrm{Ar}}\right)$, $124.2(C(5)), 122.4\left(\mathrm{C}_{\mathrm{Ar}}\right), 119.9(C(6)), 109.6\left(\mathrm{HC}_{\mathrm{Ar}}\right), 46.9$ $\left(\mathrm{PhCH} \mathrm{H}_{2} \mathrm{R}\right), 17.6\left(\mathrm{RCH}_{3}\right)$.

2-(1-Benzyl-2-(2-methyl-1,3-dioxolan-2-yl)-1H-indol-3-yl)1-phenylethan-1-one (15). This compound was prepared according to general procedure $\mathrm{A}$ on $0.15 \mathrm{mmol}$ scale, affording 15 (50 mg, 84\%) as an off-white solid. Mp: 128 $131{ }^{\circ} \mathrm{C}$. IR $v_{\max }$ (thin film) 3058, 3029, 2989, 2891, 1691, 1197, $742 \mathrm{~cm}^{-1}$. HRMS: calculated for $\mathrm{C}_{27} \mathrm{H}_{25} \mathrm{O}_{3} \mathrm{NNa}$, 434.17266 [M+Na $]^{+}$, found $\mathrm{m} / \mathrm{z}, 434.17270, \Delta=0.08 \mathrm{ppm} .{ }^{1} \mathrm{H}$ NMR $\left(400 \mathrm{MHz}, \mathrm{CDCl}_{3}\right) \delta_{\square}: 8.15-8.11\left(2 \mathrm{H}, \mathrm{m}, 2 \times \mathrm{HC}_{\mathrm{Ar}}\right)$, 7.63-7.56 $\left(1 \mathrm{H}, \mathrm{m}, H \mathrm{C}_{\mathrm{Ar}}\right), 7.55-7.46\left(3 \mathrm{H}, \mathrm{m}, 3 \times H \mathrm{C}_{\mathrm{Ar}}\right), 7.30-$ $7.12\left(6 \mathrm{H}, \mathrm{m}, 6 \times H \mathrm{C}_{\mathrm{Ar}}\right), 6.98-6.93\left(2 \mathrm{H}, \mathrm{m}, 2 \times H \mathrm{C}_{\mathrm{Ar}}, 5.67(2 \mathrm{H}\right.$, $\left.\mathrm{s}, \mathrm{PhCH}_{2} \mathrm{~N}\right), 4.72\left(2 \mathrm{H}, \mathrm{s}, \mathrm{ArCH}_{2} \mathrm{C}(\mathrm{O})\right), 3.88-3.80(2 \mathrm{H}, \mathrm{m}$ $\left.\mathrm{CH}_{a} \mathrm{H}_{\mathrm{b}} \mathrm{CH}_{a} \mathrm{H}_{\mathrm{b}}\right), 3.68-3.62\left(2 \mathrm{H}, \mathrm{m}, \mathrm{CH}_{\mathrm{a}} H_{\mathrm{b}} \mathrm{CH}_{\mathrm{a}} H_{\mathrm{b}}\right), 1.65(3 \mathrm{H}, \mathrm{s}$, $\left.\mathrm{CCH}_{3}\right) ;{ }^{13} \mathrm{C} \mathrm{NMR}\left(100 \mathrm{MHz}, \mathrm{CDCl}_{3}\right) \delta_{\mathrm{C}}: 198.2$ (C(O)), 139.0,137.5, 137.4, $136.6\left(4 \times C_{\mathrm{Ar}}\right), 132.8,128.6,128.5(3 \times$ $\left.\mathrm{H} C_{\mathrm{Ar}}\right), 128.4\left(C_{\mathrm{Ar}}\right), 128.3,126.7,125.8,122.5,119.8,118.6$, $\left.110.3\left(7 \times \mathrm{HC}_{\mathrm{Ar}}\right), 107.3,106.8\left(C_{\mathrm{Ar}}+\mathrm{CCH}_{3}\right), 64.8\left(\mathrm{OCH}_{2}\right)_{2}\right)$, $48.0\left(\mathrm{PhCH}_{2} \mathrm{~N}\right), 35.2\left(\mathrm{ArCH} \mathrm{H}_{2} \mathrm{C}(\mathrm{O})\right), 27.2\left(\mathrm{CCH}_{3}\right)$.

9-Benzyl-1-methyl-3-phenyl-9H-pyrido[3,4-b]indole (16). This compound was prepared according to a modification of general procedure B on a $85 \mu \mathrm{mol}$ scale, affording $16(28 \mathrm{mg}$, $94 \%)$ as a brown solid. A mixture of $\mathrm{EtOH} / \mathrm{H}_{2} \mathrm{O} / \mathrm{DMF}(3: 1: 2)$ was used as solvent and the temperature was kept at $110{ }^{\circ} \mathrm{C}$ for this cyclization. Mp: $127-129{ }^{\circ} \mathrm{C}$. IR $v_{\max }$ (thin film) 3060 , 3030, 1472, 734, $694 \mathrm{~cm}^{-1}$. HRMS: calculated for $\mathrm{C}_{25} \mathrm{H}_{21} \mathrm{~N}_{2}$, $349.16993[\mathrm{M}+\mathrm{H}]^{+}$, found $\mathrm{m} / z$ 349.16943, $\Delta=-1.41 \mathrm{ppm} .{ }^{1} \mathrm{H}$ NMR (500 MHz, $\left.\mathrm{CDCl}_{3}\right) \delta_{\square}: 8.31(1 \mathrm{H}, \mathrm{s}, H \mathrm{C}(4)), 8.22(1 \mathrm{H}, \mathrm{d}$, $\left.J=7.7 \mathrm{~Hz}, H \mathrm{C}_{\mathrm{Ar}}\right), 8.16-8.12\left(2 \mathrm{H}, \mathrm{m}, 2 \times H \mathrm{C}_{\mathrm{Ar}}\right), 7.56-7.48$ $\left(3 \mathrm{H}, \mathrm{m}, 3 \times H \mathrm{C}_{\mathrm{Ar}}\right), 7.41-7.35\left(2 \mathrm{H}, \mathrm{m}, 2 \times H \mathrm{C}_{\mathrm{Ar}}\right), 7.34-7.22$ $\left(4 \mathrm{H}, \mathrm{m}, 5 \times H \mathrm{C}_{\mathrm{Ar}}\right), 7.05-7.00\left(2 \mathrm{H}, \mathrm{m}, 2 \times H \mathrm{C}_{\mathrm{Ar}}\right), 5.81(2 \mathrm{H}, \mathrm{s}$, $\left.\mathrm{PhCH}_{2} \mathrm{~N}\right), 2.95\left(3 \mathrm{H}, \mathrm{s}, \mathrm{CH}_{3}\right) ;{ }^{13} \mathrm{C}$ NMR $\left(125 \mathrm{MHz} \mathrm{CDCl}_{3}\right) \delta_{\mathrm{C}}$ : $145.9,141.3,140.2,139.4,137.0,133.9,129.2\left(7 \times C_{\mathrm{Ar}}\right)$, $127.9,127.6,127.3,126.5,126.5,125.7,124.4\left(7 \times \mathrm{H} C_{\mathrm{Ar}}\right)$, $120.7\left(C_{\mathrm{Ar}}\right), 120.4,119.0,108.9\left(3 \times \mathrm{HC}_{\mathrm{Ar}}\right), 108.5(C(4)), 47.2$ $\left(\mathrm{PhCH}_{2} \mathrm{~N}\right), 22.4\left(\mathrm{CH}_{3}\right)$.

2-(2-Benzoyl-1-benzyl-1H-indol-3-yl)-1-phenylethan-1-one (17). This compound was prepared according to general procedure A on a $6.36 \mathrm{mmol}$ scale, affording $17(1.75 \mathrm{~g}, 64 \%)$ as a yellow solid. Mp: $132-134{ }^{\circ} \mathrm{C}$. IR $v_{\max }$ (thin film) 3059 , 3030, 1689, 1637, 745, $729 \mathrm{~cm}^{-1}$. HRMS: calculated for $\mathrm{C}_{30} \mathrm{H}_{23} \mathrm{O}_{2} \mathrm{NNa}, 452.16210[\mathrm{M}+\mathrm{Na}]^{+}$, found $\mathrm{m} / \mathrm{z} 452.16183, \Delta=$ -0.59 ppm. ${ }^{1} \mathrm{H}$ NMR $\left(400 \mathrm{MHz}, \mathrm{CDCl}_{3}\right) \delta_{\square}: 7.78(2 \mathrm{H}, \mathrm{ddd}, J$ $\left.=8.0,1.3,0.9 \mathrm{~Hz}, 2 \times H \mathrm{C}_{\mathrm{Ar}}\right), 7.74-7.69\left(2 \mathrm{H}, \mathrm{m}, 2 \times H \mathrm{C}_{\mathrm{Ar}}\right)$, $7.58\left(1 \mathrm{H}, \mathrm{d}, J=8.0 \mathrm{~Hz}, H \mathrm{C}_{\mathrm{Ar}}\right), 7.54-7.48\left(1 \mathrm{H}, \mathrm{m}, H \mathrm{C}_{\mathrm{Ar}}\right), 7.43-$ $7.13\left(11 \mathrm{H}, \mathrm{m}, 11 \times H \mathrm{C}_{\mathrm{Ar}}\right), 7.05-7.00\left(2 \mathrm{H}, \mathrm{m}, 2 \times H \mathrm{C}_{\mathrm{Ar}}\right), 5.59$ $\left(2 \mathrm{H}, \mathrm{s}, \mathrm{PhCH}_{2} \mathrm{Ar}\right), 4.26\left(2 \mathrm{H}, \mathrm{s}, \mathrm{CH}_{2} \mathrm{C}(\mathrm{O})\right) ;{ }^{13} \mathrm{C} \mathrm{NMR}(100$ $\left.\mathrm{MHz}, \mathrm{CDCl}_{3}\right) \delta_{\mathrm{C}}: 196.4,190.2(2 \times C(\mathrm{O})), 139.6,138.8$, $138.0,136.6,134.5\left(5 \times C_{\mathrm{Ar}}\right), 133.0,132.8,129.4,128.6$, 
128.6, 128.5, $128.1\left(7 \times \mathrm{HC}_{\mathrm{Ar}}\right), 127.5\left(C_{\mathrm{Ar}}\right), 127.3,126.5$, 125.6, 121.1, $120.9\left(5 \times \mathrm{H} C_{\mathrm{Ar}}\right), 116.1\left(C_{\mathrm{Ar}}\right), 110.9\left(\mathrm{H} C_{\mathrm{Ar}}\right)$, $48.1\left(\mathrm{PhCH} \mathrm{H}_{2} \mathrm{Ar}\right), 36.0\left(\mathrm{CH}_{2} \mathrm{C}(\mathrm{O})\right)$.

9-Benzyl-1,3-diphenyl-9H-pyrido[3,4-b]indole (18). This compound was prepared according to a modification of general procedure $\mathrm{B}$ on a $0.17 \mathrm{mmol}$ scale, affording 18 (61 $\mathrm{mg}, 85 \%$ ) as a yellow solid. A mixture of $\mathrm{EtOH} / \mathrm{H}_{2} \mathrm{O} / \mathrm{DMF}$ (3:1:2) was used as solvent and the temperature was kept at $110{ }^{\circ} \mathrm{C}$ for this cyclization. Mp: $170-174{ }^{\circ} \mathrm{C}$. IR $v_{\max }$ (thin film) 3058, 3031, 1468, 1450, 737, $694 \mathrm{~cm}^{-1}$. HRMS: calculated for $\mathrm{C}_{30} \mathrm{H}_{22} \mathrm{~N}_{2}, 411.18558[\mathrm{M}+\mathrm{H}]^{+}$, found $\mathrm{m} / \mathrm{z}$ 411.18452, $\Delta=-2.57$ ppm. ${ }^{1} \mathrm{H}$ NMR $\left(400 \mathrm{MHz}, \mathrm{CDCl}_{3}\right) \delta_{\square}$ : $8.48(1 \mathrm{H}, \mathrm{s}, H \mathrm{C}(4)), 8.30(1 \mathrm{H}, \mathrm{ddd}, J=7.8,1.2,0.8 \mathrm{~Hz}$, $\left.H \mathrm{C}_{\mathrm{Ar}}\right), 8.23-8.18\left(2 \mathrm{H}, \mathrm{m}, 2 \times H_{\mathrm{Ar}}\right), 7.58-7.47(5 \mathrm{H}, \mathrm{m}, 5 \times$ $\left.H \mathrm{C}_{\mathrm{Ar}}\right), 7.45-7.30\left(6 \mathrm{H}, \mathrm{m}, 6 \times H_{\mathrm{Ar}}\right), 7.20-7.10(3 \mathrm{H}, \mathrm{m}, 3 \times$ $\left.H \mathrm{C}_{\mathrm{Ar}}\right), 6.68-6.62\left(2 \mathrm{H}, \mathrm{m}, 2 \times \mathrm{HC}_{\mathrm{Ar}}\right), 5.25\left(2 \mathrm{H}, \mathrm{s}, \mathrm{PhCH}_{2} \mathrm{~N}\right)$; ${ }^{13} \mathrm{C}$ NMR $\left(100 \mathrm{MHz}, \mathrm{CDCl}_{3}\right) \delta_{\mathrm{C}}: 147.1,144.0,143.2,140.3$, $139.9,137.1,134.0,131.8\left(8 \times C_{\mathrm{Ar}}\right), 129.6,128.7,128.6$, $128.4,128.3,128.0,127.8,127.1,127.0,125.8\left(10 \times \mathrm{H} C_{\mathrm{Ar}}\right)$, $122.0\left(C_{\mathrm{Ar}}\right), 121.6,120.3\left(2 \times \mathrm{H} C_{\mathrm{Ar}}\right), 110.8(C(3)), 110.2$ $\left(\mathrm{H} C_{\mathrm{Ar}}\right), 48.2\left(\mathrm{PhCH}_{2} \mathrm{~N}\right)$.

9-Benzyl-3-(tert-butyl)-9H-pyrido[3,4-b]indole (19). This compound was prepared according to general procedure D, affording 19 (51 mg, 53\%) as a brown solid. Mp: 132 - 134 ${ }^{\circ} \mathrm{C}$. IR $v_{\max }$ (thin film) 3030, 2956, 2864, 1468, 740, $698 \mathrm{~cm}^{-1}$. HRMS: calculated for $\mathrm{C}_{22} \mathrm{H}_{23} \mathrm{~N}_{2}, 315.18558[\mathrm{M}+\mathrm{H}]^{+}$, found $m / z$ 315.18530, $\Delta=-0.86 \mathrm{ppm} .{ }^{1} \mathrm{H}$ NMR $\left(400 \mathrm{MHz}, \mathrm{CDCl}_{3}\right) \delta$ : $8.82(1 \mathrm{H}, \mathrm{d}, J=0.9 \mathrm{~Hz}, H \mathrm{C}(1)), 8.19(1 \mathrm{H}, \mathrm{d}, J=7.8 \mathrm{~Hz}$, $\left.H \mathrm{C}_{\mathrm{Ar}}\right), 8.19(1 \mathrm{H}, \mathrm{d}, J=0.9 \mathrm{~Hz}, H \mathrm{C}(4)), 7.55(1 \mathrm{H}, \mathrm{ddd}, J=8.3$, $\left.7.1,1.2 \mathrm{~Hz}, H \mathrm{C}_{\mathrm{Ar}}\right), 7.43\left(1 \mathrm{H}, \mathrm{d}, J=8.3 \mathrm{~Hz}, H \mathrm{C}_{\mathrm{Ar}}\right), 7.32-7.18$ $\left(6 \mathrm{H}, \mathrm{m}, 6 \times \mathrm{HC}_{\mathrm{Ar}}\right), 5.53\left(2 \mathrm{H}, \mathrm{s}, \mathrm{PhCH}_{2} \mathrm{~N}\right), 1.51(9 \mathrm{H}, \mathrm{s}$, $\left.\left(\mathrm{CH}_{3}\right)_{3} \mathrm{C}\right) ;{ }^{13} \mathrm{C} \mathrm{NMR}\left(100 \mathrm{MHz}, \mathrm{CDCl}_{3}\right) \delta_{\mathrm{C}}: 159.0,141.8$, $136.7,135.0\left(4 \times C_{\mathrm{Ar}}\right), 130.8(C(1)), 129.3\left(C_{\mathrm{Ar}}\right), 128.9,128.2$, 127.8, 126.7, $121.8\left(5 \times \mathrm{H} C_{\mathrm{Ar}}\right), 121.6\left(C_{\mathrm{Ar}}\right), 119.6,109.6(2 \times$ $\left.\mathrm{H}_{\mathrm{Ar}}\right), 109.4((4)), 47.1\left(\mathrm{PhCH}_{2} \mathrm{~N}\right), 37.2\left(\left(\mathrm{CH}_{3}\right)_{3} C\right), 30.9$ $\left(\left(\mathrm{CH}_{3}\right)_{3} \mathrm{C}\right)$.

9-Benzyl-4-isopropyl-3-phenyl-9H-pyrido[3,4-b]indole (20). This compound was prepared according to general procedure D, affording 20 (84 mg, 73\%) as a brown solid. Mp: 170 - 171 ${ }^{\circ} \mathrm{C}$. IR $v_{\max }$ (thin film) 3055, 3030, 2990, 2958, 2929, 2872, 1439, 741, $701 \mathrm{~cm}^{-1}$. HRMS: calculated for $\mathrm{C}_{27} \mathrm{H}_{24} \mathrm{~N}_{2}$, $377.20123[\mathrm{M}+\mathrm{H}]^{+}$, found $\mathrm{m} / z$ 377.20139, $\Delta=0.42 \mathrm{ppm} .{ }^{1} \mathrm{H}$ NMR (500 MHz, $\left.\mathrm{CDCl}_{3}\right) \delta_{\square}: 8.89(1 \mathrm{H}, \mathrm{s}, \mathrm{HC}(1)), 7.62-7.53$ $\left(3 \mathrm{H}, \mathrm{m}, 3 \times \mathrm{HC}_{\mathrm{Ar}}\right), 7.46-7.37\left(4 \mathrm{H}, \mathrm{m}, 4 \times H \mathrm{C}_{\mathrm{Ar}}\right), 7.33-7.23$ $\left(5 \mathrm{H}, \mathrm{m}, 5 \times H \mathrm{C}_{\mathrm{Ar}}\right), 6.95\left(1 \mathrm{H}, \mathrm{dd}, J=8.0,6.9 \mathrm{~Hz}, H \mathrm{C}_{\mathrm{Ar}}\right), 6.83$ $\left(1 \mathrm{H}, \mathrm{d}, J=8.0 \mathrm{~Hz}, H \mathrm{C}_{\mathrm{Ar}}\right) 5.53\left(2 \mathrm{H}, \mathrm{s}, \mathrm{PhCH}_{2} \mathrm{~N}\right), 3.18(1 \mathrm{H}$, hept, $\left.J=6.8 \mathrm{~Hz}, \mathrm{CH}\left(\mathrm{CH}_{3}\right)_{2}\right), 1.29(6 \mathrm{H}, \mathrm{d}, J=6.8 \mathrm{~Hz}$, $\left.\mathrm{CH}\left(\mathrm{CH}_{3}\right)_{2}\right) ;{ }^{13} \mathrm{C} \mathrm{NMR}\left(100 \mathrm{MHz}, \mathrm{CDCl}_{3}\right) \delta_{\mathrm{C}}: 153.9,141.8$, 138.6, 136.7, $134.9\left(5 \times C_{\mathrm{Ar}}\right), 130.9,129.3,128.9,128.9(4 \times$ $\left.\mathrm{H} C_{\mathrm{Ar}}\right), 128.9128 .8\left(2 \times C_{\mathrm{Ar}}\right), 127.8,127.8,127.8,126.8$, $123.5\left(5 \times \mathrm{H} C_{\mathrm{Ar}}\right), 121.6\left(C_{\mathrm{Ar}}\right), 119.4,109.3\left(2 \times \mathrm{H} C_{\mathrm{Ar}}\right), 47.0$ $\left(\mathrm{PhCH}_{2} \mathrm{~N}\right), 30.9\left(\mathrm{CH}\left(\mathrm{CH}_{3}\right)_{2}\right), 23.2\left(\mathrm{CH}\left(\mathrm{CH}_{3}\right)_{2}\right)$.

9-benzyl-3-(furan-2-yl)-4-methyl-9H-pyrido[3,4-b]indole (21). This compound was prepared according to general procedure D, affording 21 (78 $\mathrm{mg}, 75 \%)$ as a brown solid. Mp: $156-159{ }^{\circ} \mathrm{C}$. IR $v_{\max }$ (thin film) $3141,2918,1488,1301,733$, $696 \mathrm{~cm}^{-1}$. HRMS: calculated for $\mathrm{C}_{23} \mathrm{H}_{19} \mathrm{ON}_{2}, 339.14919$ $[\mathrm{M}+\mathrm{H}]^{+}$, found $m / z, 339.14883, \Delta=-1.05 \mathrm{ppm} .{ }^{1} \mathrm{H}$ NMR $(500$ $\left.\mathrm{MHz}, \mathrm{CDCl}_{3}\right) \delta_{\square}: 8.63(1 \mathrm{H}, \mathrm{s}, H \mathrm{C}(1)), 8.19(1 \mathrm{H}, \mathrm{d}, J=8.0$
$\left.\mathrm{Hz}, H \mathrm{C}_{\mathrm{Ar}}\right), 7.50(1 \mathrm{H}, \mathrm{dd}, J=1.9,0.7 \mathrm{~Hz}, \mathrm{OC} H), 7.44(1 \mathrm{H}$, $\left.\mathrm{ddd}, J=8.2,7.2,1.1 \mathrm{~Hz}, H \mathrm{C}_{\mathrm{Ar}}\right), 7.33(1 \mathrm{H}, \mathrm{d}, J=8.3 \mathrm{~Hz}$, $\left.H \mathrm{C}_{\mathrm{Ar}}\right), 7.20\left(1 \mathrm{H}\right.$, ddd, $\left.J=8.0,7.3,0.6 \mathrm{~Hz}, H \mathrm{C}_{\mathrm{Ar}}\right), 7.16-7.09$ $\left(3 \mathrm{H}, \mathrm{m}, 3 \times H \mathrm{C}_{\mathrm{Ar}}\right), 7.03-6.99\left(2 \mathrm{H}, \mathrm{m}, 2 \times H \mathrm{C}_{\mathrm{Ar}}\right), 6.67(1 \mathrm{H}, \mathrm{dd}$, $J=3.3,0.7 \mathrm{~Hz}, \mathrm{OCCH}), 6.46(1 \mathrm{H}, \mathrm{dd}, J=3.3,1.9 \mathrm{~Hz}$, $\mathrm{OCHCH}), 5.39\left(2 \mathrm{H}, \mathrm{s}, \mathrm{PhCH}_{2} \mathrm{~N}\right), 2.89\left(3 \mathrm{H}, \mathrm{s}, \mathrm{CH}_{3}\right) ;{ }^{13} \mathrm{C} \mathrm{NMR}$ $\left(125 \mathrm{MHz}, \mathrm{CDCl}_{3}\right) \delta_{\mathrm{C}}: 154.1\left(C_{\mathrm{Ar}}\right), 142.2(\mathrm{OCH}), 141.6$, $139.1,136.4,135.3\left(4 \times C_{\mathrm{Ar}}\right), 129.6(C(1)), 128.9\left(\mathrm{H} C_{\mathrm{Ar}}\right)$, $128.3\left(C_{\mathrm{Ar}}\right), 127.8,127.8,126.5\left(3 \times \mathrm{H}_{\mathrm{Ar}}\right), 125.4\left(C_{\mathrm{Ar}}\right), 124.2$ $\left(\mathrm{H} C_{\mathrm{Ar}}\right), 122.4\left(C_{\mathrm{Ar}}\right), 120.1\left(\mathrm{H} C_{\mathrm{Ar}}\right), 111.2(\mathrm{OCHCH}), 109.7$ $\left(\mathrm{HC}_{\mathrm{Ar}}\right), 109.6(\mathrm{OCCH}), 46.8\left(\mathrm{PhCH}_{2} \mathrm{~N}\right), 16.8\left(\mathrm{CH}_{3}\right)$.

9-Benzyl-4-ethyl-3-phenyl-9H-pyrido[3,4-b]indole (22). This compound was prepared according to general procedure D, affording 22 (76 mg, 68\%) as a brown solid. Mp: 136 - 138 ${ }^{\circ} \mathrm{C}$. IR $v_{\max }$ (thin film) 3056, 2969, 2933, 2873, 1441, 730, 700 $\mathrm{cm}^{-1}$. HRMS: calculated for $\mathrm{C}_{26} \mathrm{H}_{23} \mathrm{~N}_{2}, 363.18558[\mathrm{M}+\mathrm{H}]^{+}$, found $\mathrm{m} / \mathrm{z}$ 363.18530, $\Delta=-0.75 \mathrm{ppm} .{ }^{1} \mathrm{H} \mathrm{NMR}(500 \mathrm{MHz}$, $\left.\mathrm{CDCl}_{3}\right) \delta_{\square}: 8.81(1 \mathrm{H}, \mathrm{s}, C(1)), 8.28\left(1 \mathrm{H}, \mathrm{d}, J=8.0 \mathrm{~Hz}, H \mathrm{C}_{\mathrm{Ar}}\right)$, 7.61-7.55 (3H, m, $\left.3 \times H \mathrm{C}_{\mathrm{Ar}}\right), 7.52-7.46\left(3 \mathrm{H}, \mathrm{m}, 3 \times H \mathrm{C}_{\mathrm{Ar}}\right)$, 7.44-7.39 $\left(1 \mathrm{H}, \mathrm{m}, H \mathrm{C}_{\mathrm{Ar}}\right), 7.35(1 \mathrm{H}, \mathrm{ddd}, J=8.0,7.1,1.0 \mathrm{~Hz}$, $\left.H \mathrm{C}_{\mathrm{Ar}}\right), 7.32-7.26\left(3 \mathrm{H}, \mathrm{m}, 3 \times \mathrm{CC}_{\mathrm{Ar}}\right), 7.24-7.20(2 \mathrm{H}, \mathrm{m}, 2 \times$ $\left.H \mathrm{C}_{\mathrm{Ar}}\right), 5.60\left(2 \mathrm{H}, \mathrm{s}, \mathrm{PhCH}_{2} \mathrm{~N}\right), 3.26(2 \mathrm{H}, \mathrm{q}, J=7.5 \mathrm{~Hz}$, $\left.\mathrm{CH}_{2} \mathrm{CH}_{3}\right), 1.44\left(3 \mathrm{H}, \mathrm{t}, \mathrm{J}=7.5 \mathrm{~Hz}, \mathrm{CH}_{2} \mathrm{CH}_{3}\right) ;{ }^{13} \mathrm{C} \mathrm{NMR}(125$ $\left.\mathrm{MHz}, \mathrm{CDCl}_{3}\right) \delta_{\mathrm{C}}: 149.1,141.7,141.5,136.6,136.2131 .2(6 \times$ $\left.C_{\mathrm{Ar}}\right), 129.5(C(1)), 129.4,129.0,128.1,127.8,127.8(5 \times$ $\left.\mathrm{H} C_{\mathrm{Ar}}\right), 127.4\left(C_{\mathrm{Ar}}\right), 127.2,126.6,124.2\left(3 \times \mathrm{H} C_{\mathrm{Ar}}\right), 121.5$ $\left(C_{\mathrm{Ar}}\right), \quad 120.1, \quad 109.7\left(2 \times \mathrm{HC}_{\mathrm{Ar}}\right), \quad 46.9\left(\mathrm{PhCH}_{2} \mathrm{~N}\right), \quad 23.4$ $\left(\mathrm{CH}_{2} \mathrm{CH}_{3}\right), 14.5\left(\mathrm{CH}_{2} \mathrm{CH}_{3}\right)$.

9-Benzyl-3-(2-methoxyphenyl)-9H-pyrido[3,4-b]indole (23) This compound was prepared according to general procedure D, affording 23 (83 mg, 74\%) as a yellow solid. Mp: 95 - 97 ${ }^{\circ} \mathrm{C}$. IR $v_{\max }$ (thin film) $3030,2935,2834,1491,727,700 \mathrm{~cm}^{-1}$. HRMS: calculated for $\mathrm{C}_{25} \mathrm{H}_{20} \mathrm{~N}_{2} \mathrm{O}, 365.16484[\mathrm{M}+\mathrm{H}]^{+}$, found $m / z, 365.16464, \Delta=-0.54 \mathrm{ppm} .{ }^{1} \mathrm{H}$ NMR $\left(500 \mathrm{MHz}, \mathrm{CDCl}_{3}\right) \delta$ $\square: 8.94(1 \mathrm{H}, \mathrm{d}, J=1.0 \mathrm{~Hz}, H \mathrm{C}(1)), 8.51(1 \mathrm{H}, \mathrm{d}, J=1.0 \mathrm{~Hz}$, $H C(4)), 8.22\left(1 \mathrm{H}\right.$, ddd, $\left.J=7.8,1.2,0.7 \mathrm{~Hz}, H \mathrm{C}_{\mathrm{Ar}}\right), 7.84,(1 \mathrm{H}$, $\left.\mathrm{dd}, J=7.6,1.8 \mathrm{~Hz}, H \mathrm{C}_{\mathrm{Ar}}\right), 7.57(1 \mathrm{H}, \mathrm{ddd}, J=8.3,7.1,1.2 \mathrm{~Hz}$, $\left.H \mathrm{C}_{\mathrm{Ar}}\right), 7.46-7.42\left(1 \mathrm{H}, \mathrm{m}, H \mathrm{C}_{\mathrm{Ar}}\right), 7.38(1 \mathrm{H}, \mathrm{ddd}, \mathrm{J}=8.3,7.4$, $\left.1.8 \mathrm{~Hz}, H \mathrm{C}_{\mathrm{Ar}}\right), 7.34-7.18\left(6 \mathrm{H}, \mathrm{m}, 6 \times H \mathrm{C}_{\mathrm{Ar}}\right), 7.14-7.09(1 \mathrm{H}, \mathrm{m}$, $\left.H \mathrm{C}_{\mathrm{Ar}}\right), 7.06\left(1 \mathrm{H}, \mathrm{dd}, \mathrm{J}=8.3,1.0 \mathrm{~Hz}, H \mathrm{C}_{\mathrm{Ar}}\right), 5.58(2 \mathrm{H}, \mathrm{s}$, $\left.\mathrm{PhCH}_{2} \mathrm{~N}\right), 3.91\left(3 \mathrm{H}, \mathrm{s}, \mathrm{CH}_{3} \mathrm{O}\right) ;{ }^{13} \mathrm{C}$ NMR $\left(125 \mathrm{MHz}, \mathrm{CDCl}_{3}\right) \delta$ C: $156.8,146.0,141.8,136.6,135.7\left(5 \times C_{\mathrm{Ar}}\right), 131.6\left(\mathrm{HC}_{\mathrm{Ar}}\right)$, $131.5(C(1)), 130.2,129.0\left(2 \times C_{\mathrm{Ar}}\right), 128.9,128.9,128.4$, 127.8, 126.7, $122.0\left(6 \times \mathrm{HC}_{\mathrm{Ar}}\right), 121.7\left(C_{\mathrm{Ar}}\right), 121.1,119.8(2 \times$ $\left.\mathrm{H} C_{\mathrm{Ar}}\right), 115.9(C(4)), 111.5,109.7\left(2 \times \mathrm{HC}_{\mathrm{Ar}}\right), 55.8\left(C \mathrm{H}_{3} \mathrm{O}\right)$, $47.0\left(\mathrm{PhCH}_{2} \mathrm{~N}\right)$.

3-((3r,5r,7r)-Adamantan-1-yl)-9-benzyl-9H-pyrido[3,4-

b]indole (24). This compound was prepared according to general procedure D, affording $24(73 \mathrm{mg}, 61 \%)$ as a brown solid. Mp: $241-243{ }^{\circ} \mathrm{C}$. IR $v_{\max }$ (thin film) 2901, 2846, 1464, 1452, 738, $698 \mathrm{~cm}^{-1}$. HRMS: calculated for $\mathrm{C}_{28} \mathrm{H}_{30} \mathrm{~N}_{2}$, $393.23253[\mathrm{M}+\mathrm{H}]^{+}$, found $m / z .393 .23257, \Delta=0.12 \mathrm{ppm} .{ }^{1} \mathrm{H}$ NMR $\left(500 \mathrm{MHz}, \mathrm{CDCl}_{3}\right) \delta_{\square}: 8.82(1 \mathrm{H}, \mathrm{d}, J=1.0 \mathrm{~Hz}, H C(1))$, $8.17\left(1 \mathrm{H}, \mathrm{d}, J=7.8 \mathrm{~Hz}, H \mathrm{C}_{\mathrm{Ar}}\right), 7.96(1 \mathrm{H}, \mathrm{d}, J=1.0 \mathrm{~Hz}$, $H C(4)), 7.54\left(1 \mathrm{H}\right.$, ddd, $\left.J=8.3,7.2,1.1 \mathrm{~Hz}, H \mathrm{C}_{\mathrm{Ar}}\right), 7.43(1 \mathrm{H}$, $\left.\mathrm{d}, J=8.3 \mathrm{~Hz}, H \mathrm{C}_{\mathrm{Ar}}\right), 7.30-7.23\left(4 \mathrm{H}, \mathrm{m}, 4 \times H_{\mathrm{Ar}}\right), 7.22-7.18$ $\left(2 \mathrm{H}, \mathrm{m}, 2 \times \mathrm{HC}_{\mathrm{Ar}}\right), 5.53\left(2 \mathrm{H}, \mathrm{s}, \mathrm{PhCH}_{2} \mathrm{~N}\right), 2.19-2.15(3 \mathrm{H}, \mathrm{m}$, $3 \times \mathrm{CH}), 2.14\left(6 \mathrm{H}, \mathrm{d}, J=2.9 \mathrm{~Hz}, \mathrm{RC}\left(\mathrm{CH}_{2}\right)_{3}\right), 1.84(6 \mathrm{H}, \mathrm{t}, J=$ $\left.3.1,3 \times \mathrm{CH}_{2}\right) ;{ }^{13} \mathrm{C} \mathrm{NMR}\left(125 \mathrm{MHz}, \mathrm{CDCl}_{3}\right) \delta_{\mathrm{C}}: 158.0,140.7$, 
$135.7,134.0\left(4 \times C_{\mathrm{Ar}}\right), 129.9(C(1)), 128.3\left(C_{\mathrm{Ar}}\right), 127.8,127.1$, 126.7, 125.7, $120.7\left(5 \times \mathrm{H} C_{\mathrm{Ar}}\right), 120.6\left(C_{\mathrm{Ar}}\right), 118.5\left(\mathrm{H} C_{\mathrm{Ar}}\right)$, $108.5(C(4)), 108.2\left(\mathrm{HC}_{\mathrm{Ar}}\right), 46.0\left(\mathrm{PhCH}_{2} \mathrm{~N}\right), 41.6\left(\mathrm{RC}\left(\mathrm{CH}_{2}\right)_{3}\right)$, $37.6\left(\mathrm{RC}\left(\mathrm{CH}_{2}\right)_{3}\right), 35.9\left(\mathrm{CH}_{2}\right), 28.0(\mathrm{CH})$.

9-Benzyl-3-(4-fluorophenyl)-4-methyl-9H-pyrido[3,4-

b]indole (25). This compound was prepared according to general procedure D, affording $25(58 \mathrm{mg}, 52 \%)$ as a brown solid. Mp: 183 - $185{ }^{\circ} \mathrm{C}$. IR $v_{\max }$ (thin film) 3056, 14551219 , 731, $699 \mathrm{~cm}^{-1}$. HRMS: calculated for $\mathrm{C}_{25} \mathrm{H}_{19} \mathrm{FN}_{2}, 367.16050$ $[\mathrm{M}+\mathrm{H}]^{+}$, found $\mathrm{m} / \mathrm{z}$ 367.16034, $\Delta=-0.45 \mathrm{ppm} .{ }^{1} \mathrm{H}$ NMR $(500$ $\left.\mathrm{MHz}, \mathrm{CDCl}_{3}\right) \delta_{\square}: 8.78(1 \mathrm{H}, \mathrm{s}, H \mathrm{C}(1)), 8.32(1 \mathrm{H}, \mathrm{d}, J=7.8$ $\left.\mathrm{Hz}, H \mathrm{C}_{\mathrm{Ar}}\right), 7.62-7.54\left(3 \mathrm{H}, \mathrm{m}, 3 \times H \mathrm{C}_{\mathrm{Ar}}\right), 7.49(1 \mathrm{H}, \mathrm{d}, J=8.2$, $\left.H \mathrm{C}_{\mathrm{Ar}}\right), 7.35\left(1 \mathrm{H}, \mathrm{t}, J=7.6 \mathrm{~Hz}, H \mathrm{C}_{\mathrm{Ar}}\right), 7.37-7.24(3 \mathrm{H}, \mathrm{m}, 3 \times$ $\left.H \mathrm{C}_{\mathrm{Ar}}\right), 7.21-7.14\left(4 \mathrm{H}, \mathrm{m}, 4 \times \mathrm{HC}_{\mathrm{Ar}}\right), 5.59\left(2 \mathrm{H}, \mathrm{s}, \mathrm{PhCH}_{2} \mathrm{~N}\right)$, $2.89\left(3 \mathrm{H}, \mathrm{s}, \mathrm{CH}_{3}\right) ;{ }^{13} \mathrm{C}$ NMR $\left(125 \mathrm{MHz}, \mathrm{CDCl}_{3}\right) \delta_{\mathrm{C}}: 161.2(\mathrm{~d}$, $J=246 \mathrm{~Hz}, C \mathrm{~F}), 147.0,140.6\left(2 \times C_{\mathrm{Ar}}\right), 136.1(\mathrm{~d}, J=3.3 \mathrm{~Hz}$, CCHCHCF $), 135.4,134.6\left(2 \times C_{\mathrm{Ar}}\right) 130.5(\mathrm{~d}, J=8.1 \mathrm{~Hz}$, $C H C F), 128.2\left(\mathrm{H}_{\mathrm{Ar}}\right), 127.9(\mathrm{HC}(1)), 127.4\left(C_{\mathrm{Ar}}\right), 126.8(\mathrm{~d}, J$ $=6.2 \mathrm{~Hz}, C \mathrm{HCHF}), 125.5\left(\mathrm{H} C_{\mathrm{Ar}}\right), 123.8\left(C_{\mathrm{Ar}}\right), 123.1\left(\mathrm{H} C_{\mathrm{Ar}}\right)$, $121.2\left(C_{\mathrm{Ar}}\right), 118.9,114.0,113.8,108.6\left(4 \times \mathrm{HC}_{\mathrm{Ar}}\right), 45.8$ $\left(\mathrm{PhCH}_{2} \mathrm{~N}\right), 16.5\left(\mathrm{CH}_{3}\right) ;{ }^{19} \mathrm{~F}$ NMR $\left(376 \mathrm{MHz}, \mathrm{CDCl}_{3}\right) \delta_{\mathrm{F}}: 115.4$ (tt, $J=8.8,5.5 \mathrm{~Hz})$.

3-(Benzo[d][1,3]dioxol-5-yl)-9-benzyl-4-methyl-9Hpyrido[3,4-b]indole (26). This compound was prepared according to general procedure D, affording 26 (71 $\mathrm{mg}, 61 \%)$ as a brown solid. Mp: $152-155{ }^{\circ} \mathrm{C}$. IR $v_{\max }$ (thin film) 2890, 1459, $725 \mathrm{~cm}^{-1}$. HRMS: calculated for $\mathrm{C}_{25} \mathrm{H}_{19} \mathrm{~N}_{2}, 379.14410$ $[\mathrm{M}+\mathrm{H}]^{+}$, found $m / z$ 379.14352, $\Delta=-1.53 \mathrm{ppm} .{ }^{1} \mathrm{H}$ NMR $(500$ $\left.\mathrm{MHz}, \mathrm{CDCl}_{3}\right) \delta_{\square}: 8.84(1 \mathrm{H}, \mathrm{d}, J=0.9 \mathrm{~Hz}, H \mathrm{C}(1)), 8.30(1 \mathrm{H}$, $\mathrm{d}, J=0.9 \mathrm{~Hz}, H \mathrm{C}(4)), 8.20\left(1 \mathrm{H}, \mathrm{d}, J=7.8 \mathrm{~Hz}, H \mathrm{C}_{\mathrm{Ar}}\right), 7.64-$ $7.54\left(3 \mathrm{H}, \mathrm{m}, 3 \times H \mathrm{C}_{\mathrm{Ar}}\right), 7.43\left(1 \mathrm{H}, \mathrm{d}, J=8.3 \mathrm{~Hz}, H \mathrm{C}_{\mathrm{Ar}}\right), 7.34-$ $7.22\left(4 \mathrm{H}, \mathrm{m}, 4 \times H_{\mathrm{Ar}}\right), 7.20-7.16\left(2 \mathrm{H}, \mathrm{m}, 2 \times H \mathrm{C}_{\mathrm{Ar}}\right), 6.94$ $\left(1 \mathrm{H}, \mathrm{d}, J=8.1 \mathrm{~Hz}, H_{\mathrm{Ar}}\right), 6.01\left(2 \mathrm{H}, \mathrm{s}, \mathrm{O}_{2} \mathrm{CH}_{2}\right), 5.54(2 \mathrm{H}, \mathrm{s}$, $\left.\mathrm{PhCH}_{2} \mathrm{~N}\right) ;{ }^{13} \mathrm{C} \mathrm{NMR}\left(125 \mathrm{MHz}, \mathrm{CDCl}_{3}\right) \delta_{\mathrm{C}}: 148.2,147.5$, $147.5,141.9,136.5,135.8,135.1\left(7 \times C_{\mathrm{Ar}}\right), 131.6(\mathrm{HC}(1))$, $129.8\left(C_{\mathrm{Ar}}\right), 129.0,128.6,127.9,126.6,121.9\left(5 \times \mathrm{H} C_{\mathrm{Ar}}\right)$, $121.6\left(C_{\mathrm{Ar}}\right), 120.4,120.0\left(2 \times \mathrm{HC}_{\mathrm{Ar}}\right), 110.8(\mathrm{HC}(4)), 109.8$, 108.5, $107.4\left(3 \times \mathrm{HC}_{\mathrm{Ar}}\right), 101.2\left(\mathrm{O}_{2} \mathrm{CH}_{2}\right), 47.0\left(\mathrm{PhCH}_{2} \mathrm{~N}\right)$.

Synthesis of 2-(1-benzyl-2-(1,3-dioxolan-2-yl)-1H-indol3-yl)-1,3-diphenylpropan-1-one (27). A fresh solution of LiHMDS was prepared in a dry vial adding sequentially dry THF (7.3 mL), HMDS (290 $\mu \mathrm{L}, 1.39 \mathrm{mmol}, 2.5$ eq.), a $2.5 \mathrm{M}$ solution of $n \mathrm{BuLi}(1.39 \mathrm{mmol}, 558 \mu \mathrm{L}, 2.5$ eq.) and stirring at $-78{ }^{\circ} \mathrm{C}$ for 10 minutes. Acetophenone $(130 \mu \mathrm{L}, 1.07 \mathrm{mmol}, 2$ eq.) was then added at $0{ }^{\circ} \mathrm{C}$ and stirred for 15 minutes. In a second dry vial were added bromo indole 1 (200 mg, 558 $\mu \mathrm{mol})$ and $\mathrm{Pd}(\mathrm{d} t \mathrm{bpf}) \mathrm{Cl}_{2}(18 \mathrm{mg}, 27.9 \mu \mathrm{mol}, 5 \mathrm{~mol} \%)$. The flask was sealed, evacuated and backfilled with argon twice. The freshly formed enolate solution was then transferred via syringe to the flask. The mixture was stirred at $50{ }^{\circ} \mathrm{C}$ for 24 hours in an oil bath. After cooling to room temperature, benzyl bromide (166 $\mu \mathrm{L}, 1.39 \mathrm{mmol}, 2.5$ eq.) was added and the mixture was stirred at $90{ }^{\circ} \mathrm{C}$ for $24 \mathrm{~h}$. The resulting mixture was filtered through a plug of silica and concentrated in vacuo. The crude product was purified by flash column chromatography (eluted with 9:1 petroleum ether/EtOAc) to afford product $27(177 \mathrm{mg}, 65 \%)$ as a brown solid. Mp: 134 $135^{\circ} \mathrm{C}$. IR $v_{\max }$ (thin film) $3060,3027,2924,1679,1083,742$, $697 \mathrm{~cm}^{-1}$. HRMS: calculated for $\mathrm{C}_{33} \mathrm{H}_{30} \mathrm{O}_{3} \mathrm{~N}, 488.22202$
$[\mathrm{M}+\mathrm{H}]^{+}$, found $m / z$ 488.22195, $\Delta=-0.14 \mathrm{ppm} .{ }^{1} \mathrm{H}$ NMR $(400$ $\left.\mathrm{MHz}, \mathrm{C}_{6} \mathrm{D}_{6}\right) \delta_{\square}: 8.24\left(2 \mathrm{H}, \mathrm{ddd}, J=8.2,1.9,1.5 \mathrm{~Hz}, H \mathrm{C}_{\mathrm{Ar}}\right)$, $8.05\left(1 \mathrm{H}, \mathrm{d}, J=8.05 \mathrm{~Hz}, H \mathrm{C}_{\mathrm{Ar}}\right), 7.17-7.14\left(1 \mathrm{H}, \mathrm{m}, H \mathrm{C}_{\mathrm{Ar}}\right)$, $7.12-6.89\left(12 \mathrm{H}, \mathrm{m}, H \mathrm{C}_{\mathrm{Ar}}\right), 6.86\left(1 \mathrm{H}, \mathrm{d}, J=8.2 \mathrm{~Hz}, H \mathrm{C}_{\mathrm{Ar}}\right), 6.72$ $(2 \mathrm{H}, \mathrm{ddd}, J=7.6,1.9,1.3 \mathrm{~Hz}, 2 \times H \mathrm{CAr}), 5.92(1 \mathrm{H}, \mathrm{s}$, $\left.\mathrm{CH}(\mathrm{OR})_{2}\right), 5.39\left(1 \mathrm{H}, \mathrm{dd}, J=8.4,6.0 \mathrm{~Hz}, \mathrm{CH}_{2} \mathrm{CHC}(\mathrm{O})\right), 5.15$ $\left(1 \mathrm{H}, \mathrm{d}, J=17.1 \mathrm{~Hz}, \mathrm{PhCH}_{a} \mathrm{H}_{\mathrm{b}} \mathrm{Ar}\right), 5.09(1 \mathrm{H}, \mathrm{d}, J=17.1 \mathrm{~Hz}$, $\left.\mathrm{PhCH}_{\mathrm{a}} H_{b} \mathrm{Ar}\right), 3.99\left(1 \mathrm{H}, \mathrm{dd}, J=13.5,6.0 \mathrm{~Hz}, \mathrm{PhCH}_{a} \mathrm{H}_{\mathrm{b}} \mathrm{CH}\right)$, $3.59\left(1 \mathrm{H}, \mathrm{dd}, J=13.5,8.4 \mathrm{~Hz}, \mathrm{PhCH}_{\mathrm{a}} \mathrm{CH}_{b} \mathrm{CH}\right), 3.36-3.13(4 \mathrm{H}$, $\left.\mathrm{m},\left(\mathrm{OCH}_{2}\right)_{2}\right) ;{ }^{13} \mathrm{C} \mathrm{NMR}\left(100 \mathrm{MHz}, \mathrm{C}_{6} \mathrm{D}_{6}\right) \delta_{\mathrm{C}}: 198.9(\mathrm{C}(\mathrm{O}))$, $140.8,138.3,137.6,137.3\left(4 \times C_{\mathrm{Ar}}\right), 132.0\left(\mathrm{H} C_{\mathrm{Ar}}\right), 129.9$, $\left(C_{\mathrm{Ar}}\right), 129.4,128.8,128.3,128.0,128.0,126.7\left(6 \times \mathrm{H} C_{\mathrm{Ar}}\right)$, $126.3\left(C_{\mathrm{Ar}}\right), 125.9,125.8,123.1,120.5,120.3\left(5 \times \mathrm{H}_{\mathrm{Ar}}\right)$, $113.9 \quad\left(C_{\mathrm{Ar}}\right), \quad 110.3 \quad\left(\mathrm{H} C_{\mathrm{Ar}}\right), \quad 99.5 \quad\left(C \mathrm{H}(\mathrm{OR})_{2}\right), \quad 64.6$ $\left(\mathrm{O}_{2} \mathrm{C}_{a} \mathrm{H}_{2} \mathrm{C}_{\mathrm{b}} \mathrm{H}_{2}\right), 64.3\left(\mathrm{O}_{2} \mathrm{C}_{\mathrm{a}} \mathrm{H}_{2} C_{\mathrm{b}} \mathrm{H}_{2}\right), 48.0\left(\mathrm{CH}_{2} \mathrm{CHC}(\mathrm{O})\right), 47.5$ $\left(\mathrm{PhCH} \mathrm{H}_{2} \mathrm{Ar}\right), 38.2\left(\mathrm{CH}_{2} \mathrm{CH}\right)$

4,9-Dibenzyl-3-phenyl-9H-pyrido[3,4-b]indole (28). This compound was prepared according to general procedure $\mathrm{B}$ on a $0.18 \mathrm{mmol}$ scale, affording 28 (75 $\mathrm{mg}, 96 \%)$ as an off-white solid. Mp: $201-204{ }^{\circ} \mathrm{C}$. IR $v_{\max }$ (thin film) 3056, 3026, 1448, 740, 728, $699 \mathrm{~cm}^{-1}$. HRMS: calculated for $\mathrm{C}_{31} \mathrm{H}_{25} \mathrm{~N}_{2}$, $425.20123[\mathrm{M}+\mathrm{H}]^{+}$, found $\mathrm{m} / \mathrm{z}$ 425.20102, $\Delta=-0.48 \mathrm{ppm} .{ }^{1} \mathrm{H}$ NMR (400 MHz, CDCl $)) \delta_{\square}: 8.95(1 \mathrm{H}, \mathrm{s}, H C(1)), 7.89(1 \mathrm{H}, \mathrm{d}$, $\left.J=8.0 \mathrm{~Hz}, H \mathrm{C}_{\mathrm{Ar}}\right), 7.55-7.44\left(4 \mathrm{H}, \mathrm{m}, 4 \times H \mathrm{C}_{\mathrm{Ar}}\right), 7.42-7.10$ $\left(14 \mathrm{H}, \mathrm{m}, 14 \times \mathrm{HC}_{\mathrm{Ar}}\right), 5.64\left(2 \mathrm{H}, \mathrm{s}, \mathrm{PhCH}_{2} \mathrm{~N}\right), 4.68(2 \mathrm{H}, \mathrm{s}$, $\left.\mathrm{PhCH}_{2} \mathrm{Ar}\right) ;{ }^{13} \mathrm{C} \mathrm{NMR}\left(100 \mathrm{MHz}, \mathrm{CDCl}_{3}\right) \delta_{\mathrm{C}}: 150.3,141.8$, 141.0, 139.7, 136.5, $136.0\left(6 \times C_{\mathrm{Ar}}\right), 130.3(\mathrm{HC}(1)), 129.5$, $129.0\left(2 \times \mathrm{HC}_{\mathrm{Ar}}\right), 128.9\left(C_{\mathrm{Ar}}\right), 128.7,128.2,128.0,128.0$, $127.9,127.4,126.7,126.1\left(8 \times \mathrm{H}_{\mathrm{Ar}}\right), 126.0\left(C_{\mathrm{Ar}}\right), 124.4$ $\left(\mathrm{H} C_{\mathrm{Ar}}\right), 121.5\left(C_{\mathrm{Ar}}\right), 120.1,109.6\left(2 \times \mathrm{HC}_{\mathrm{Ar}}\right), 47.0\left(\mathrm{PhCH}_{2} \mathrm{~N}\right)$, $36.4\left(\mathrm{PhCH} \mathrm{H}_{2} \mathrm{Ar}\right)$.

4-Allyl-9-benzyl-3-phenyl-9H-pyrido[3,4-b]indole (29). This compound was prepared according to general procedure F, affording 29 (62 mg, 54\%) as an off-white solid. Mp: 156 $158{ }^{\circ} \mathrm{C}$. IR $v_{\max }$ (thin film) 3056, 2926, 1487, 739, $700 \mathrm{~cm}^{-1}$. HRMS: calculated for $\mathrm{C}_{27} \mathrm{H}_{23} \mathrm{~N}_{2}, 375.18558[\mathrm{M}+\mathrm{H}]^{+}$, found $m / z$ 375.18552, $\Delta=-0.16$ ppm. ${ }^{1} \mathrm{H}$ NMR (400 MHz, $\left.\mathrm{CDCl}_{3}\right) \delta$ : $8.75(1 \mathrm{H}, \mathrm{s}, H \mathrm{C}(1)), 8.09\left(1 \mathrm{H}, \mathrm{d}, J=8.0 \mathrm{~Hz}, H \mathrm{C}_{\mathrm{Ar}}\right), 7.52$ $\left(2 \mathrm{H}, \mathrm{d}, J=7.0 \mathrm{~Hz}, 2 \times H \mathrm{C}_{\mathrm{Ar}}\right), 7.44\left(1 \mathrm{H}, \mathrm{t}, J=7.5 \mathrm{~Hz}, H \mathrm{C}_{\mathrm{Ar}}\right)$, 7.39-7.25 $\left(4 \mathrm{H}, \mathrm{m}, 4 \times H \mathrm{C}_{\mathrm{Ar}}\right), 7.23-7.05\left(6 \mathrm{H}, \mathrm{m}, 6 \times H \mathrm{C}_{\mathrm{Ar}}\right)$, 6.23-6.10 (1H, m, $\left.\mathrm{ArCH}_{2} \mathrm{CHCH}_{2}\right), 5.48\left(2 \mathrm{H}, \mathrm{s}, \mathrm{PhCH}_{2} \mathrm{~N}\right), 5.08$ $\left(1 \mathrm{H}, \mathrm{dd}, J=10.3,1.3 \mathrm{~Hz}, \mathrm{CH}_{c i s} \mathrm{H}_{\text {trans }} \mathrm{CHAr}\right), 4.87$ (1H, dd, $J=$ 17, $\left.1.3 \mathrm{~Hz}, \quad \mathrm{CH}_{\text {cis }} H_{\text {trans }} \mathrm{CHAr}\right), \quad 3.92-3.84 \quad(2 \mathrm{H}, \mathrm{m}$, $\left.\mathrm{ArCH}_{2} \mathrm{CHCH}_{2}\right) ;{ }^{13} \mathrm{C}$ NMR $\left(100 \mathrm{MHz}, \mathrm{CDCl}_{3}\right) \delta_{\mathrm{C}}: 149.8$, $141.8,141.1,136.5,135.9\left(5 \times C_{\mathrm{Ar}}\right), 135.6\left(\mathrm{ArCH}_{2} \mathrm{CHCH}_{2}\right)$, $130.0,129.5,129.0\left(3 \times \mathrm{HC}_{\mathrm{Ar}}\right), 128.4\left(C_{\mathrm{Ar}}\right), 128.0,128.0$, $127.9,127.4,126.6\left(5 \times \mathrm{HC}_{\mathrm{Ar}}\right), 125.9\left(C_{\mathrm{Ar}}\right), 124.6\left(\mathrm{H} C_{\mathrm{Ar}}\right)$, $121.4 \quad\left(\mathrm{C}_{\mathrm{Ar}}\right), 120.0\left(\mathrm{HC}_{\mathrm{Ar}}\right), 116.8 \quad\left(\mathrm{ArCH}_{2} \mathrm{CHCH}_{2}\right), 109.7$ $\left(\mathrm{HC}_{\mathrm{Ar}}\right), 47.0\left(\mathrm{PhCH}_{2} \mathrm{~N}\right), 34.5\left(\mathrm{ArCH}_{2} \mathrm{CHCH}_{2}\right)$.

9-Benzyl-4-(4-methoxybenzyl)-3-phenyl-9H-pyrido[3,4b]indole (30). This compound was prepared according to general procedure $\mathrm{F}$, affording $30(74 \mathrm{mg}, 53 \%)$ as a yellow solid. Mp: $208-209{ }^{\circ} \mathrm{C}$. IR $v_{\max }$ (thin film) 3058, 2932, 2835, 1244, 735, $699 \mathrm{~cm}^{-1}$. HRMS: calculated for $\mathrm{C}_{32} \mathrm{H}_{26} \mathrm{~N}_{2} \mathrm{O}$, $455.21179[\mathrm{M}+\mathrm{H}]^{+}$, found $\mathrm{m} / \mathrm{z}$ 455.21158, $\Delta=-0.46 \mathrm{ppm} .{ }^{1} \mathrm{H}$ NMR (400 MHz, $\left.\mathrm{CDCl}_{3}\right) \delta_{\square}: 8.80(1 \mathrm{H}, \mathrm{s}, H \mathrm{C}(1)), 7.78(1 \mathrm{H}$, $\left.\mathrm{d}, J=8.0 \mathrm{~Hz}, H \mathrm{C}_{\mathrm{Ar}}\right), 7.42-7.30\left(4 \mathrm{H}, \mathrm{m}, 4 \times H \mathrm{C}_{\mathrm{Ar}}\right), 7.28-7.08$ $\left(8 \mathrm{H}, \mathrm{m}, 8 \times H \mathrm{C}_{\mathrm{Ar}}\right), 7.05-6.98\left(1 \mathrm{H}, \mathrm{m}, H \mathrm{C}_{\mathrm{Ar}}\right), 6.94(2 \mathrm{H}, \mathrm{d}, J=$ $\left.8.6 \mathrm{~Hz}, 2 \times H \mathrm{C}_{\mathrm{Ar}}\right), 6.70-6.65\left(2 \mathrm{H}, \mathrm{m}, 2 \times H \mathrm{C}_{\mathrm{Ar}}\right), 5.49(2 \mathrm{H}, \mathrm{s}$, 
$\left.\mathrm{PhCH}_{2} \mathrm{~N}\right), 4.47\left(2 \mathrm{H}, \mathrm{s}, \mathrm{PMBCH} \mathrm{H}_{2} \mathrm{Ar}\right), 3.63\left(3 \mathrm{H}, \mathrm{s}, \mathrm{CH}_{3} \mathrm{O}\right) ;{ }^{13} \mathrm{C}$ NMR $\left(100 \mathrm{MHz}, \mathrm{CDCl}_{3}\right) \delta_{\mathrm{C}}: 157.9,150.2,141.8,141.0$, $136.5,136.0,131.7\left(7 \times C_{\mathrm{Ar}}\right), 130.3(C(1)), 129.5,129.1$, $129.0\left(3 \times \mathrm{H}_{\mathrm{Ar}}\right), 128.9\left(C_{\mathrm{Ar}}\right), 128.0,128.0,127.9,127.4$, $126.7\left(5 \times \mathrm{H} C_{\mathrm{Ar}}\right), 126.4\left(C_{\mathrm{Ar}}\right), 124.5\left(\mathrm{H} C_{\mathrm{Ar}}\right), 121.5\left(C_{\mathrm{Ar}}\right)$, 120.1, 114.1, $109.6\left(3 \times \mathrm{HC}_{\mathrm{Ar}}\right), 55.2\left(\mathrm{CH}_{3} \mathrm{O}\right), 47.0\left(\mathrm{PhCH}_{2} \mathrm{~N}\right)$, $35.6\left(\mathrm{PMBC} \mathrm{H}_{2} \mathrm{Ar}\right)$.

9-Benzyl-4-(naphthalen-2-ylmethyl)-3-phenyl-9H-

pyrido[3,4-b]indole (31). This compound was prepared according to general procedure $\mathrm{F}$, affording $\mathbf{3 1}$ as a brown solid (70 mg, 48\%). Mp: $181-183{ }^{\circ} \mathrm{C}$. IR $v_{\max }$ (thin film) 3052, 734, $699 \mathrm{~cm}^{-1}$. HRMS: calculated for $\mathrm{C}_{35} \mathrm{H}_{27} \mathrm{~N}_{2}$, $475.21688[\mathrm{M}+\mathrm{H}]^{+}$, found $\mathrm{m} / \mathrm{z}$ 475.21644, $\Delta=-0.91 \mathrm{ppm} .{ }^{1} \mathrm{H}$ NMR (500 MHz, CDCl $)_{3} \delta_{\square}: 9.01(1 \mathrm{H}, \mathrm{s}, H C(1)), 7.88(1 \mathrm{H}, \mathrm{d}$, $\left.J=8.1 \mathrm{~Hz}, H \mathrm{C}_{\mathrm{Ar}}\right), 7.85-7.81\left(2 \mathrm{H}, \mathrm{m}, 2 \times H \mathrm{C}_{\mathrm{Ar}}\right), 7.61(1 \mathrm{H}, \mathrm{d}, J$ $\left.=7.8 \mathrm{~Hz}, H \mathrm{C}_{\mathrm{Ar}}\right), 7.57-7.52\left(2 \mathrm{H}, \mathrm{m}, 2 \times H \mathrm{C}_{\mathrm{Ar}}\right), 7.51-7.46(3 \mathrm{H}$, $\left.\mathrm{m}, 3 \times H \mathrm{C}_{\mathrm{Ar}}\right), 7.45-7.25\left(11 \mathrm{H}, \mathrm{m}, 11 \times H \mathrm{C}_{\mathrm{Ar}}\right), 7.09-7.03(1 \mathrm{H}$, $\left.\mathrm{m}, \mathrm{HC}_{\mathrm{Ar}}\right), 5.66\left(2 \mathrm{H}, \mathrm{s}, \mathrm{PhCH}_{2} \mathrm{~N}\right), 4.84\left(2 \mathrm{H}, \mathrm{s}, \mathrm{C}_{10} \mathrm{H}_{7} \mathrm{CH}_{2} \mathrm{Ar}\right)$; ${ }^{13} \mathrm{C}$ NMR $\left(125 \mathrm{MHz}, \mathrm{CDCl}_{3}\right) \delta_{\mathrm{C}}: 150.3,141.9,140.9,137.4$, $136.5,136.1,133.8,132.2\left(8 \times C_{\mathrm{Ar}}\right), 130.5(C(1)), 129.5$, $129.0\left(2 \times \mathrm{H}_{\mathrm{Ar}}\right), 129.0\left(C_{\mathrm{Ar}}\right), 128.5,128.1,128.0,127.9$, $127.8,127.6,127.5,127.0,126.7,126.3,126.0\left(11 \times \mathrm{H} C_{\mathrm{Ar}}\right)$, $125.8\left(C_{\mathrm{Ar}}\right), 125.4,124.4\left(2 \times \mathrm{H}_{\mathrm{Ar}}\right), 121.5\left(C_{\mathrm{Ar}}\right), 120.5,109.6$ $\left(2 \times \mathrm{HC}_{\mathrm{Ar}}\right), 47.1\left(\mathrm{PhCH}_{2} \mathrm{~N}\right), 36.8\left(\mathrm{C}_{10} \mathrm{H}_{7} \mathrm{CH}_{2} \mathrm{Ar}\right)$.

Acknowledgment. We thank CAPES, Coordenação de Aperfeiçoamento de Pessoal de Nível Superior, Brazil and AstraZeneca for supporting this project.

Supporting Information Available: Spectroscopic data for all new compounds are available. This material is available free of charge via the Internet at http://pubs.acs.org.

(1) (a) Freedland, C. S.; Mansbach, R. S. Drug Alcohol Depend. 1999, 54, 183. (b) Hassani, M.; Cal, W.; Koelsch, K. H.; Holley, D. C.; Rose, A. S.; Olang, F.; Lineswala, J. P.; Hollaway, W. G.; Gerdes, J. M.; Behforouz, M.; Beall, H. D. J. Med. Chem. 2008, 51, 3104. (c) Dai, J.-K.; Dan, W.-J.; Li, N.; Du, H.-T.; Zhang, J.-W.; Wang, J.-R. Bioorg. Chem. Lett. 2016 26, 580. (d) Frédérick, R.; Bruyère, C.; Vancraeynest, C.; Reniers, J.; Meinguet, C.; Pochet, L.; Backlung, A.; Masereel, B.; Kiss, R.; Wouters, J. J. Med. Chem. 2012, 55, 6489.

(2) Sun, B.; Morikawa, T.; Matsuda, H.; Tewtrakul, S.; Wu, L. J.; Harima, S.; Yoshikawa, M. J. Nat. Prod. 2004, 67, 1464.

(3) Jaeger, R. J. R.; Lambshöft, M.; Gottfried, S.; Spiteller, M.; Spiteller, P. J. Nat. Prod. 2013, 76, 127.

(4) Xin, W.-B.; Chou, G.-X.; Wang, Z.-T. Phytochem. Lett. 2011, 4, 380.

(5) Inman, W. D.; Bray, W. M.; Gassner, N. C.; Lokey, R. S.; Tenney, K.; Shen, Y. Y.; TenDyke, K.; Suh, T.; Crews, P. J. Nat. Prod. 2010, 73, 255. (6) Tran, T. D.; Pham, N. B.; Ekins, M.; Hooper, J. N. A.; Quinn, R. J. Mar. Drugs 2015, 13, 4556.

(7) (a) Too, P. C.; Chua, S. H.; Wong, S. H.; Chiba, S. J. Org. Chem. 2011, 76, 6159. (b) Ding, S.; Shi, Z.; Jiao, N. Org. Lett. 2010, 12, 1540. (c) Zhang, H.; Larock, C. J. Org. Chem. 2002, 67, 9318.

(8) Nissen, F.; Richard, V.; Alayrac, C.; Witulski, B. Chem. Commun. 2011, 47, 6656 .

(9) Dhiman, S.; Mishra, U.K.; Ramasastry, S. S. V. Angew. Chem. Int. Ed. 2016, $55,7737$.

(10) Laha, J. K.; Barolo, S. M.; Rossi, R. A.; Cuny, G. D. J. Org. Chem. 2011, 76, 6421.

(11) Namjoshi, O. A.; Gryboski, A.; Cooj, J. M. J. Org. Chem. 2011, 76, 4721.
(12) Chiba, S.; Xu, Y.-J.; Wang, Y.-F. J. Am. Chem. Soc. 2009, 131, 12886.

(13) Kamlah, A.; Lirk, F.; Bracher, F. Tetrahedron 2016, 72, 837-845.

(14) (a) Hamann, B. C.; Hartwig, J. F. J. Am. Chem. Soc. 1997, 119, 11108-11109. (b) Palucki, M.; Buchwald, S. L. J. Am. Chem. Soc. 1997 119, 12382-12383. (c) Satoh, T.; Kawamura, Y.; Miura, M.; Nomura, M. Angew. Chem. Int. Ed. 1997, 36, 1740-1742. For reviews, see: (d) Bellina, F.; Rossi, R. Chem. Rev. 2010, 110, 1082. (e) Johansson, C. C. C.; Colacot, T. J. Angew. Chem. Int. Ed. 2010, 49, 676.

(15) Potukuchi, H. K.; Spork, A. P.; Donohoe, T. J. Org. Biomol. Chem. 2015, 13, 4367.

(16) (a) Donohoe, T. J.; Pilgrim, B. S.; Jones, C. R.; Bassuto, J. A. Proc. Natl. Acad. Sci. US 2012, 109, 11605. (b) Pilgrim, B.; Gatland, A. E.; McTernan, C.; Procopiou, P.; Donohoe, T. J. Org. Lett. 2013, 15, 6190. (c) Gatland, A. E.; Pilgrim, B. S.; Procopiou, P. A.; Donohoe, T. J. Angew. Chem. Int. Ed. 2014, 53, 14555. (d) Pilgrim, B. S.; Esteves, C. H. A.; McTernan, C. T.; Jones, G. R.; Tatton, M. R.; Procopiou, P. A.; Donohoe, T. J. Org. Biomol. Chem. 2016, 14, 1065.

(17) Hardegger, L. A.; Habegger, J.; Donohoe, T. J. Org. Lett. 2015, 17, 3222 .

(18) Solé, D.; Bennasar, M.-L.; Jiménez, I. Org. Biomol. Chem. 2011, 9, $4535-4544$

(19) (a) Beare, N. A.; Hartwig, J. .F. J. Org. Chem. 2002, 67, 541. (b) Si, C.; Myers, A. G. Angew. Chem. Int. Ed. 2011, 50, 10409.

(20) Biswas, S.; Batra, S. Adv. Synth. Catal. 2011, 353, 2861-2867.

(21) Kawatsure, M.; Hartwig, J. F. J. Am. Chem. Soc. 1999, 121, 1473.

(22) Suzuki, H.; Iwata, C.; Sakurai, K.; Tokumoto, K.; Takahashi, H.; Hanada, M.; Yokoyama, Y.; Murakami, Y. Tetrahedron 1997, 53, 15931606.

(23) Kolhatkar, R. B.; Ghorai, S. K.; George, C.; Reith, M. E. A.; Dutta, A. K. J. Med. Chem. 2003, 46, 2205-2215.

(24) Benson, S. C.; Gross, J. L.; Snyder, J. K. J. Org. Chem. 1990, 55, 3257-3269.

(25) Sechi, M.; Derudas, M.; Dallocchio, R.; Dessì, A.; Bacchi, A.; Sannia, L.; Carta, F.; Palomba, M.; Ragab, O.; Chan, C.; Shoemaker, R.; Sei, S.; Dayam, R.; Neamati, N. J. Med. Chem. 2004, 47, 5298-5310. (26) Miki, Y.; Hachiken, H.; Yoshikawa, I. Heterocycles 1997, 45, $1143-$ 1150 . 